\title{
A Dual-Mixed Approximation Method for a Three-Field Model of a Nonlinear Generalized Stokes Problem
}

\author{
Vincent J. Ervin* Jason S. Howell ${ }^{\dagger} \quad$ Iuliana Stanculescu ${ }^{\ddagger}$
}

September 12, 2007

\begin{abstract}
In this work a dual-mixed approximation of a nonlinear generalized Stokes problem is studied. The problem is analyzed in Sobolev spaces which arise naturally in the problem formulation. Existence and uniqueness results are given and error estimates are derived. It is shown that both lowest-order and higher-order mixed finite elements are suitable for the approximation method. Numerical experiments that support the theoretical results are presented.
\end{abstract}

Key words. generalized Stokes problem, dual-mixed method, twofold saddle point problem, Sobolev spaces

\section{Introduction}

In this article we investigate the solution of a nonlinear generalized Stokes problem using a dualmixed formulation. The nonlinear generalized Stokes problem arises in modeling flows of, for example, biological fluids, lubricants, paints, polymeric fluids, where the fluid viscosity is assumed to be a nonlinear function of the fluid's velocity gradient tensor. The generalized Stokes problem is given by: Find $(\mathbf{u}, p)$ such that

$$
\begin{aligned}
-\nabla \cdot(\nu(|\nabla \mathbf{u}|) \nabla \mathbf{u})+\nabla p & =\mathbf{f} \quad \text { in } \Omega \\
\nabla \cdot \mathbf{u} & =0 \quad \text { in } \Omega, \\
\mathbf{u} & =\mathbf{u}_{\Gamma} \text { on } \Gamma,
\end{aligned}
$$

where $\Omega$ is a bounded open subset of $\mathbb{R}^{n}$ with Lipschitz continuous boundary $\Gamma$. The fluid velocity is denoted by $\mathbf{u}$, and $\nabla \mathbf{u}:=(\nabla \mathbf{u})_{i j}=\partial u_{i} / \partial x_{j}$ is the tensor gradient of $\mathbf{u}$. Here and throughout the paper we use the following notation: for tensors $\boldsymbol{\sigma}=\left(\sigma_{i j}\right), \boldsymbol{\tau}=\left(\tau_{i j}\right), \boldsymbol{\sigma}: \boldsymbol{\tau}=\sum_{i, j} \sigma_{i j} \tau_{i j}$, $|\boldsymbol{\sigma}|^{2}=\boldsymbol{\sigma}: \boldsymbol{\sigma}$. The pressure is denoted by $p$, and $\mathbf{f}$ describes the external forces on the fluid. The function $\nu$ describes the nonlinear kinematic viscosity of the fluid.

\footnotetext{
*Department of Mathematical Sciences, Clemson University, Clemson, SC, 29634-0975, USA. email: vjervin@clemson.edu. This material is based upon work partially supported by the NSF under grant DMS-0410792.

${ }^{\dagger}$ Department of Mathematical Sciences, Carnegie Mellon University, Pittsburgh, PA, 15213-3890, USA. email: howel14@andrew.cmu.edu. This material is based upon work supported by the Center for Nonlinear Analysis (CNA) under the National Science Foundation Grant No. DMS 0635983, and by the National Science Foundation Grant No. DMS 0410792 while a graduate student in the Department of Mathematical Sciences at Clemson University.

${ }^{\ddagger}$ Department of Mathematics, University of Pittsburgh, Pittsburgh, PA, 15260, USA. email: ius1@pitt.edu.
} 
Some classical examples of $\nu$ are given by:

\section{Power Law}

$$
\nu(|\mathbf{d}(\mathbf{u})|)=\nu_{0}|\mathbf{d}(\mathbf{u})|^{r-2}, \quad \nu_{0}>0, \quad 1<r<2,
$$

where $\mathbf{d}(\mathbf{u})=\frac{1}{2}\left(\nabla \mathbf{u}+\nabla \mathbf{u}^{\mathrm{T}}\right)$ denotes the fluid deformation tensor. The power law model has been used to model the viscosity of many polymeric solutions and melts over a considerable range of shear rates $[18]$.

Ladyzhenskaya Law[21]:

$$
\nu(|\nabla \mathbf{u}|)=\left(\nu_{0}+\nu_{1}|\nabla \mathbf{u}|\right)^{r-2}, \quad \nu_{0} \geq 0, \nu_{1}>0, r>1,
$$

which has been used in modeling fluids with large stresses.

\section{Carreau Law:}

$$
\nu(|\mathbf{d}(\mathbf{u})|)=\nu_{0}\left(1+|\mathbf{d}(\mathbf{u})|^{2}\right)^{(r-2) / 2}, \quad \nu_{0}>0, r \geq 1,
$$

used in modeling visco-plastic flows and creeping flow of metals.

General descriptions of (1.1) are often written in terms of the tensor $\boldsymbol{\sigma}=\nu(|\nabla \mathbf{u}|) \nabla \mathbf{u}$ :

$$
-\nabla \cdot \boldsymbol{\sigma}+\nabla p=\mathbf{f} \quad \text { in } \Omega .
$$

The work in this paper extends the investigates of $[4,23,15]$. In [4] Baranger, Najib, and Sandri provided an analysis for the existence and uniqueness of the modeling equations in appropriate Sobolev spaces and gave an error analysis of a finite element approximation method applied to the primitive variables $(\boldsymbol{\sigma}, p, \mathbf{u})$. Manouzi and Farhloul in [23] reformulated the modeling equations into a saddle point problem and used a mixed formulation to study the existence and uniqueness of the solution, again in appropriate Sobolev spaces. An error analysis for the finite element approximation was also given. In both [4] and [23] the analysis used the assumption that the equation describing $\boldsymbol{\sigma}$ in terms of $\mathbf{d}(\mathbf{u})$ or $\nabla \mathbf{u}$ was invertible to give $\mathbf{d}(\mathbf{u})$ or $\nabla \mathbf{u}$ as a function of $\boldsymbol{\sigma}$. Gatica, González, and Meddahi in [15] reformulated the modeling equations, using the tensor $\boldsymbol{\psi}$ in place of the $\boldsymbol{\sigma}(\boldsymbol{\psi}=\boldsymbol{\sigma}-p \mathbf{I})$ and introducing an additional variable for $\nabla \mathbf{u}$. Doing so their formulation used the constitutive equation for $\boldsymbol{\sigma}$ as a function of $\nabla \mathbf{u}$ and reduced the regulatity requirement for the velocity. Advantages of this approach include: (i) more flexibility in choosing the approximating finite element space for $\mathbf{u}$, (ii) Dirichlet boundary conditions for $\mathbf{u}$ become natural boundary conditions and are easily incorporated into the variational formulations, (iii) avoids the assumption of expressing $\nabla \mathbf{u}$ was a function of $\boldsymbol{\sigma}$. A disadvantage in this formulation is that additional unknowns are introduced. The analysis of this approach was only studied in a Hilbert space setting.

In this paper we recast the formulation described in [15] in appropriate Sobolev spaces. Because of the nonlinearity in (1.7), this problem is more appropriately studied in Sobolev spaces which should result in tighter error estimates for the approximate solution. This extends the work of [23] by avoiding the assumption of expressing $\nabla \mathbf{u}$ as a function of $\boldsymbol{\sigma}$. In addition, we show that higherorder approxmiating spaces can be used in the mixed finite element method for this formulation and give the associated a priori error estimates. 
A description of the notation used in this paper, the mathematical problem, and the dual-mixed variational formulation is given in Section 2. Existence and uniqueness of the variational formulation is studied in Section 3. In Section 4 the finite element approximation is presented and analyzed. Numerical results are given in Section 5.

\section{Mathematical Setting}

For $r>1$ we denote its unitary conjugate by $r^{\prime}$, satisfying $r^{-1}+r^{\prime-1}=1$. Used in the analysis below are the following function spaces and norms.

$$
T:=\left(L^{r}(\Omega)\right)^{n \times n}=\left\{\boldsymbol{\tau}=\left(\tau_{i j}\right) ; \tau_{i j} \in L^{r}(\Omega) ; i, j=1, \ldots, n\right\},
$$

with norm $\|\boldsymbol{\tau}\|_{T}:=\left(\int_{\Omega}|\boldsymbol{\tau}|^{r} d \Omega\right)^{1 / r}$.

$$
T^{\prime}:=\left(L^{r^{\prime}}(\Omega)\right)^{n \times n} \text { and } T_{d i v}^{\prime}:=\left\{\boldsymbol{\tau} \in T^{\prime} ; \operatorname{div} \boldsymbol{\tau} \in\left(L^{r^{\prime}}(\Omega)\right)^{n}\right\}
$$

with norm $\|\boldsymbol{\tau}\|_{T_{d i v}^{\prime}}:=\left(\int_{\Omega}\left(|\boldsymbol{\tau}|^{r^{\prime}}+|\operatorname{div} \boldsymbol{\tau}|^{r^{\prime}}\right) d \Omega\right)^{1 / r^{\prime}}$. Let $U:=\left(L^{r}(\Omega)\right)^{n}$, and $P:=L^{r^{\prime}}(\Omega)$.

For a Banach space $X, X^{*}$ denotes its dual space with associated norm $\|\cdot\|_{X^{*}}$. Note that $T^{*}=T^{\prime}$, and $\left(T^{\prime}\right)^{*}=T$. The norm and seminorm associated with the Sobolev space $W^{m, r}(\Omega)$ will be denoted by $\|\cdot\|_{m, r, \Omega}$ and $|\cdot|_{m, r, \Omega}$, respectively, and the infinity norm will be denoted by $\|\cdot\|_{\infty}$.

Motivated by (1.4),(1.5),(1.6), we will assume that the extra stress tensor is a function of the velocity gradient, i.e.

$$
\boldsymbol{\sigma}:=\mathbf{g}(\nabla \mathbf{u})=\nu(|\nabla \mathbf{u}|) \nabla \mathbf{u}
$$

Specifically, we assume

A1: $\quad \mathbf{g}: T \rightarrow T^{*}$ is a bounded, continuous, strictly monotone operator [26];

and that there exist constants $\hat{C}_{1}$ and $\hat{C}_{2}$ such that, for $\mathbf{s}, \mathbf{t}, \mathbf{w} \in T$,

$$
\begin{aligned}
& \text { A2: } \int_{\Omega}(\mathbf{g}(\mathbf{s})-\mathbf{g}(\mathbf{t})):(\mathbf{s}-\mathbf{t}) d \Omega \geq \hat{C}_{1}\left(\int_{\Omega}|\mathbf{g}(\mathbf{s})-\mathbf{g}(\mathbf{t}) \| \mathbf{s}-\mathbf{t}| d \Omega+\frac{\|\mathbf{s}-\mathbf{t}\|_{T}^{2}}{\|\mathbf{s}\|_{T}^{2-r}+\|\mathbf{t}\|_{T}^{2-r}}\right), \\
& \text { A3: } \int_{\Omega}(\mathbf{g}(\mathbf{s})-\mathbf{g}(\mathbf{t})): \mathbf{w} d \Omega \leq \hat{C}_{2}\left\|\frac{|\mathbf{s}-\mathbf{t}|}{|\mathbf{s}|+|\mathbf{t}|}\right\|_{\infty}^{\frac{2-r}{r}}\left(\int_{\Omega}|\mathbf{g}(\mathbf{s})-\mathbf{g}(\mathbf{t}) \| \mathbf{s}-\mathbf{t}| d \Omega\right)^{1 / r^{\prime}}\|\mathbf{w}\|_{T},
\end{aligned}
$$

with the convention that $\mathbf{g}(\mathbf{s})=\mathbf{0}$ if $\mathbf{s}=\mathbf{0}$ and $|\mathbf{s}(\mathbf{x})-\mathbf{t}(\mathbf{x})| /(|\mathbf{s}(\mathbf{x})|+|\mathbf{t}(\mathbf{x})|)=0$ if $\mathbf{s}(\mathbf{x})=\mathbf{t}(\mathbf{x})=\mathbf{0}$. Properties A1-A3 have been established for power law and Carreau law fluids [3]. (For the case of a power law fluid monotonicity is also shown in $[28,8]$.)

Remark 2.1 From (1.2) it follows that $\mathbf{u}_{\Gamma}$ must satisfy the compatibility condition

$$
\int_{\Gamma} \mathbf{u}_{\Gamma} \cdot \mathbf{n} d \Gamma=0
$$

where $\mathbf{n}$ denotes the outward pointing unit normal vector to $\Omega$. 
In order to obtain the dual-mixed formulation, introduce two new variables, $\phi$ and $\boldsymbol{\psi}$.

$$
\begin{aligned}
\boldsymbol{\phi} & :=\nabla \mathbf{u} \\
\boldsymbol{\psi} & :=\boldsymbol{\sigma}-p \mathbf{I}, \text { the total stress tensor, } \\
& =\mathbf{g}(\boldsymbol{\phi})-p \mathbf{I}, \text { using }(2.1)
\end{aligned}
$$

With the definition of $\boldsymbol{\psi}$ a variational form for (1.1) can be written as

$$
-\int_{\Omega} \mathbf{v} \cdot \operatorname{div} \boldsymbol{\psi} d \Omega=\int_{\Omega} \mathbf{v} \cdot \mathbf{f} d \Omega, \text { for } \mathbf{v} \in T
$$

Note that from the definition of $\phi$ we have that, for sufficiently smooth functions,

$$
\begin{aligned}
0 & =-\int_{\Omega} \phi: \boldsymbol{\tau} d \Omega+\int_{\Omega} \nabla \mathbf{u}: \boldsymbol{\tau} d \Omega \\
& =-\int_{\Omega} \boldsymbol{\phi}: \boldsymbol{\tau} d \Omega+\int_{\Gamma}(\boldsymbol{\tau} \cdot \mathbf{n}) \cdot \mathbf{u}_{\Gamma} d \Gamma-\int_{\Omega} \mathbf{u} \cdot \operatorname{div} \boldsymbol{\tau} d \Omega
\end{aligned}
$$

and the condition $\operatorname{div} \mathbf{u}=0$ is equivalent to

$$
\operatorname{tr}(\phi)=0,
$$

where we use $\operatorname{tr}(\phi)$ to denote the trace of $\phi$.

Combining (1.4), (2.8), and (2.7) a variational formulation to (1.4), (2.8), and (2.7) is: Given $\mathbf{f} \in\left(L^{r^{\prime}}(\Omega)\right)^{n}, \mathbf{u}_{\Gamma} \in\left(W^{1-1 / r, r}(\Gamma)\right)^{n}$, determine $(\boldsymbol{\phi}, \boldsymbol{\psi}, p, \mathbf{u}) \in T \times T_{\text {div }}^{\prime} \times P \times U$ such that

$$
\begin{aligned}
\int_{\Omega} \mathbf{g}(\boldsymbol{\phi}): \varsigma d \Omega-\int_{\Omega} \boldsymbol{\psi}: \boldsymbol{\varsigma} d \Omega-\int_{\Omega} p \operatorname{tr}(\boldsymbol{\varsigma}) d \Omega & =0, \forall \boldsymbol{\varsigma} \in T \\
-\int_{\Omega} \boldsymbol{\tau}: \phi d \Omega-\int_{\Omega} q \operatorname{tr}(\boldsymbol{\phi}) d \Omega-\int_{\Omega} \mathbf{u} \cdot \operatorname{div} \boldsymbol{\tau} d \Omega & =-\int_{\Gamma}(\boldsymbol{\tau} \cdot \mathbf{n}) \cdot \mathbf{u}_{\Gamma} d \Gamma \\
\forall(\boldsymbol{\tau}, q) \in T_{d i v}^{\prime} \times P & \\
-\int_{\Omega} \mathbf{v} \cdot \operatorname{div} \boldsymbol{\psi} d \Omega & =\int_{\Omega} \mathbf{v} \cdot \mathbf{f} d \Omega, \forall \mathbf{v} \in U
\end{aligned}
$$

Note that equations (2.10)-(2.12) do not uniquely define a solution; as adding $(0, c \mathbf{I},-c, \mathbf{0})$ to a solution $(\boldsymbol{\phi}, \boldsymbol{\psi}, p, \mathbf{u})$, also satisfies (2.10)-(2.12) for any $c \in \mathbb{R}$. In order to guarantee uniqueness we proceed as in $[2,7,15]$ and impose, via a Lagrange multiplier, the constraint $\int_{\Omega} \operatorname{tr}(\boldsymbol{\psi}) d \Omega=0$. The variational formulation may then be restated as: Given $\mathbf{f} \in\left(L^{r^{\prime}}(\Omega)\right)^{n}, \mathbf{u}_{\Gamma} \in\left(W^{1-1 / r, r}(\Gamma)\right)^{n}$, determine $(\boldsymbol{\phi}, \boldsymbol{\psi}, p, \mathbf{u}, \lambda) \in T \times T_{\text {div }}^{\prime} \times P \times U \times \mathbb{R}$ such that

$$
\begin{aligned}
\int_{\Omega} \mathbf{g}(\boldsymbol{\phi}): \varsigma d \Omega-\int_{\Omega} \boldsymbol{\psi}: \varsigma d \Omega-\int_{\Omega} p \operatorname{tr}(\boldsymbol{\varsigma}) d \Omega & =0, \forall \boldsymbol{\varsigma} \in T \\
-\int_{\Omega} \boldsymbol{\tau}: \phi d \Omega & -\int_{\Omega} q \operatorname{tr}(\boldsymbol{\phi}) d \Omega-\int_{\Omega} \mathbf{u} \cdot \operatorname{div} \boldsymbol{\tau} d \Omega \\
=-\int_{\Gamma}(\boldsymbol{\tau} \cdot \mathbf{n}) \cdot \mathbf{u}_{\Gamma} d \Gamma, \forall(\boldsymbol{\tau}, q) \in T_{\Omega}^{\prime} \operatorname{tr}(\boldsymbol{\tau}) d \Omega & \\
-\int_{\Omega} \mathbf{v} \cdot \operatorname{div} \boldsymbol{\psi} d \Omega+\eta \int_{\Omega} \operatorname{tr}(\boldsymbol{\psi}) d \Omega & =\int_{\Omega} \mathbf{v} \cdot \mathbf{f} d \Omega, \forall(\mathbf{v}, \eta) \in U \times \mathbb{R}
\end{aligned}
$$


Remark 2.2 As commented in [15], the value of the Lagrange multiplier $\lambda$ is 0, as can be seen from the choice of $\boldsymbol{\tau}=\mathbf{I}$ and $q=-1$. However, it is included in the variational formulation so that the formulation has a twofold saddle point structure.

To formally rewrite (2.13)-(2.15) as a twofold saddle point problem define the following operators:

$$
\begin{aligned}
\mathbf{A}: T \longrightarrow T^{\prime}, \quad \mathbf{B}: T & \longrightarrow\left(T_{d i v}^{\prime} \times P\right)^{*}, \quad \mathbf{C}: T_{d i v}^{\prime} \times P \longrightarrow(U \times \mathbb{R})^{*} . \\
{[\mathbf{A}(\phi), \boldsymbol{\varsigma}] } & :=\int_{\Omega} \mathbf{g}(\boldsymbol{\phi}): \boldsymbol{\varsigma} d \Omega \\
{[\mathbf{B}(\boldsymbol{\phi}),(\boldsymbol{\tau}, q)] } & :=-\int_{\Omega} \boldsymbol{\tau}: \boldsymbol{\phi} d \Omega-\int_{\Omega} q \operatorname{tr}(\boldsymbol{\phi}) d \Omega \\
{[\mathbf{C}(\boldsymbol{\psi}, p),(\mathbf{v}, \eta)] } & :=-\int_{\Omega} \mathbf{v} \cdot \operatorname{div} \boldsymbol{\psi} d \Omega+\eta \int_{\Omega} \operatorname{tr}(\boldsymbol{\psi}) d \Omega
\end{aligned}
$$

The modeling equations can then be written in the form

$$
\begin{aligned}
{[\mathbf{A}(\phi), \boldsymbol{\varsigma}]+\left[\boldsymbol{\varsigma}, \mathbf{B}^{*}(\boldsymbol{\psi}, p)\right] } & =0, \forall \boldsymbol{\varsigma} \in T \\
{[\mathbf{B}(\phi),(\boldsymbol{\tau}, q)]+\left[(\boldsymbol{\tau}, q), \mathbf{C}^{*}(\mathbf{u}, \lambda)\right] } & =-\int_{\Gamma}(\boldsymbol{\tau} \cdot \mathbf{n}) \cdot \mathbf{u}_{\Gamma} d \Gamma, \forall(\boldsymbol{\tau}, q) \in T_{d i v}^{\prime} \times P, \\
{[\mathbf{C}(\boldsymbol{\psi}, p),(\mathbf{v}, \eta)] } & =\int_{\Omega} \mathbf{v} \cdot \mathbf{f} d \Omega, \forall(\mathbf{v}, \eta) \in U \times \mathbb{R},
\end{aligned}
$$

where $\mathbf{B}^{*}$ and $\mathbf{C}^{*}$ denote the respective adjoint operators of $\mathbf{B}$ and $\mathbf{C}$, respectively.

\section{$3 \quad$ Existence and Uniqueness}

The existence and uniqueness of (2.19)-(2.21) follows from

(i) $[\mathbf{C}(\boldsymbol{\psi}, p),(\mathbf{v}, \eta)]$ defining a bounded (componentwise) linear functional and satisfying an inf-sup condition;

(ii) $[\mathbf{B}(\phi),(\boldsymbol{\tau}, q)]$ defining a bounded (componentwise) linear functional and satisfying an inf-sup condition;

(iii) $\mathbf{A}(\phi)$ defining a bounded, continuous, strictly monotone operator on a reflexive Banach space.

Remark 3.1 A direct application of Hölder's inequality establishes that $[\mathbf{C}(\cdot, \cdot),(\cdot, \cdot)]:\left(T_{\text {div }}^{\prime} \times P\right) \times$ $(U \times \mathbb{R}) \rightarrow \mathbb{R}$ is a bounded (componentwise) linear functional.

Remark 3.2 A direct application of Hölder's inequality establishes that $[\mathbf{B}(\cdot),(\cdot, \cdot)]: T \times\left(T_{\text {div }}^{\prime} \times\right.$ $P) \rightarrow \mathbb{R}$ is a bounded (componentwise) linear functional.

Remark 3.3 From the assumptions A1-A3 discussed in Section 2, $\mathbf{A}(\boldsymbol{\phi})$ defines a bounded, continuous, strictly monotone operator on a reflexive Banach space.

Thus it remains to show the inf-sup conditions for $\mathbf{B}$ and $\mathbf{C}$. 


\subsection{Inf-sup Condition for B}

Define the null space for the operator $\mathbf{C}, Z_{1}$, as

$$
\begin{aligned}
Z_{1} & :=\left\{(\boldsymbol{\tau}, q) \in T_{d i v}^{\prime} \times P:[\mathbf{C}(\boldsymbol{\tau}, q),(\mathbf{v}, \eta)]=0, \forall(\mathbf{v}, \eta) \in U \times \mathbb{R}\right\}, \\
& =\left\{(\boldsymbol{\tau}, q) \in T_{d i v}^{\prime} \times P: \operatorname{div} \boldsymbol{\tau}=\mathbf{0} \text { in } \Omega, \text { and } \int_{\Omega} \operatorname{tr}(\boldsymbol{\tau}) d \Omega=0\right\} .
\end{aligned}
$$

Note that for $(\boldsymbol{\tau}, q) \in Z_{1},\|\boldsymbol{\tau}\|_{T_{d i v}^{\prime}}=\|\boldsymbol{\tau}\|_{T^{\prime}}$. Helpful in establishing the inf-sup condition for $\mathbf{B}$ is the following lemma.

Lemma 3.1 (See Lemma 3.1 in [2] for Hilbert space setting.)

For $\boldsymbol{\tau} \in T_{\text {div }}^{\prime}$ satisfying $\int_{\Omega} \operatorname{tr}(\boldsymbol{\tau}) d \Omega=0$, let $\boldsymbol{\tau}^{0}=\boldsymbol{\tau}-\frac{1}{n} \operatorname{tr}(\boldsymbol{\tau}) \mathbf{I}$. Then, there exists $C$, depending only $\Omega$, such that

$$
\|\boldsymbol{\tau}\|_{L^{r^{\prime}}} \leq C\left(\left\|\boldsymbol{\tau}^{0}\right\|_{L^{r^{\prime}}}+\|\operatorname{div} \boldsymbol{\tau}\|_{W^{-1, r^{\prime}}}\right) .
$$

Proof: Now, there exists a non-zero function $\varphi \in L^{r}(\Omega)$ such that

$$
\|\operatorname{tr}(\boldsymbol{\tau})\|_{L^{r^{\prime}(\Omega)}}\|\varphi\|_{L^{r}(\Omega)}=\int_{\Omega} \operatorname{tr}(\boldsymbol{\tau}) \varphi d \Omega
$$

Since $\int_{\Omega} \operatorname{tr}(\boldsymbol{\tau}) d \Omega=0$, we can assume $\int_{\Omega} \varphi d \Omega=0$ (shift $\varphi$ by its average). From [14], pg. 116, given $\varphi \in L^{r}(\Omega), 1<r<\infty$ with $\int_{\Omega} \varphi d \Omega=0$, then there exists $\mathbf{v} \in W_{0}^{1, r}(\Omega)$ and a constant $C$ such that

$$
\operatorname{div} \mathbf{v}=\varphi \text { in } \Omega \quad \text { and } \quad\|\mathbf{v}\|_{W^{1, r}(\Omega)} \leq C\|\varphi\|_{L^{r}(\Omega)}
$$

From (3.3) and (3.4),

$$
\begin{aligned}
\frac{1}{n C}\|\operatorname{tr}(\boldsymbol{\tau})\|_{L^{r^{\prime}(\Omega)}}\|\mathbf{v}\|_{W^{1, r}(\Omega)} & \leq \frac{1}{n} \int_{\Omega} \operatorname{tr}(\boldsymbol{\tau}) \operatorname{div} \mathbf{v} d \Omega=\frac{1}{n} \int_{\Omega} \operatorname{tr}(\boldsymbol{\tau}) \mathbf{I}: \nabla \mathbf{v} d \Omega \\
& \left.=\int_{\Omega}\left(\boldsymbol{\tau}-\boldsymbol{\tau}^{0}\right): \nabla \mathbf{v} d \Omega \quad \text { (using the defn. of } \boldsymbol{\tau}^{0}\right) \\
& =-\int_{\Omega}\left(\boldsymbol{\tau}^{0}: \nabla \mathbf{v}+\operatorname{div} \boldsymbol{\tau} \cdot \mathbf{v}\right) d \Omega \\
& \leq\left(\left\|\boldsymbol{\tau}^{0}\right\|_{L^{r^{\prime}}(\Omega)}+\|\operatorname{div} \boldsymbol{\tau}\|_{W^{-1, r^{\prime}}(\Omega)}\right)\|\mathbf{v}\|_{W^{1, r}(\Omega)}
\end{aligned}
$$

Lemma 3.2 There exists a constant $c_{1}>0$ such that

$$
\inf _{(\boldsymbol{\tau}, q) \in Z_{1}} \sup _{\boldsymbol{\phi} \in T} \frac{[\mathbf{B}(\boldsymbol{\phi}),(\boldsymbol{\tau}, q)]}{\|\boldsymbol{\phi}\|_{T}\|(\boldsymbol{\tau}, q)\|_{T_{d i v}^{\prime} \times P}} \geq c_{1} .
$$

Proof: We establish the inf-sup condition by considering two cases.

Case 1.: $\|q\|_{P} \leq\|\boldsymbol{\tau}\|_{T_{d i v}^{\prime}}$.

Let

$$
\boldsymbol{\tau}^{0}=\boldsymbol{\tau}-\frac{1}{n} \operatorname{tr}(\boldsymbol{\tau}) \mathbf{I}, \quad \text { and } \quad \boldsymbol{\phi}=-\left|\boldsymbol{\tau}^{0}\right|^{r^{\prime} / r-1} \boldsymbol{\tau}^{0} /\left\|\boldsymbol{\tau}^{0}\right\|_{T^{\prime}}^{r^{\prime}-1}
$$


Note that $\phi \in T$, and $\|\phi\|_{T}=1$. Then,

$$
\begin{aligned}
\frac{[B(\boldsymbol{\phi}),(\boldsymbol{\tau}, q)]}{\|\boldsymbol{\phi}\|_{T}} & =\int_{\Omega} \frac{\left|\boldsymbol{\tau}^{0}\right|^{r^{\prime} / r-1}}{\left\|\boldsymbol{\tau}^{0}\right\|_{T^{\prime}}^{r^{\prime}-1}} \boldsymbol{\tau}: \boldsymbol{\tau}^{0} d \Omega+\int_{\Omega} q \frac{|\boldsymbol{\tau}|^{r^{\prime} / r-1} \operatorname{tr}\left(\boldsymbol{\tau}^{0}\right)}{\left\|\boldsymbol{\tau}^{0}\right\|_{T^{\prime}}^{r^{\prime}-1}} d \Omega \\
& =\frac{1}{\left\|\boldsymbol{\tau}^{0}\right\|_{T^{\prime}}^{r^{\prime}-1}} \int_{\Omega}\left|\boldsymbol{\tau}^{0}\right|^{r^{\prime} / r-1} \boldsymbol{\tau}^{0}: \boldsymbol{\tau}^{0} d \Omega,\left(\text { as } \operatorname{tr}\left(\boldsymbol{\tau}^{0}\right)=0, \text { and } \boldsymbol{\tau}: \boldsymbol{\tau}^{0}=\boldsymbol{\tau}^{0}: \boldsymbol{\tau}^{0}\right) \\
& =\left\|\boldsymbol{\tau}^{0}\right\|_{T^{\prime}} \geq \frac{1}{C}\|\boldsymbol{\tau}\|_{T^{\prime}}=\frac{1}{C}\|\boldsymbol{\tau}\|_{T_{d i v}^{\prime}} \quad\left(\text { as }(\boldsymbol{\tau}, q) \in Z_{1}, \text { see }(3.2)\right) \\
& \geq \frac{1}{2 C}\left(\|\boldsymbol{\tau}\|_{T_{d i v}^{\prime}}+\|q\|_{P}\right)=\frac{1}{2 C}\|(\boldsymbol{\tau}, q)\|_{T_{d i v}^{\prime} \times P} .
\end{aligned}
$$

$\underline{\text { Case 2.: }}\|q\|_{P} \geq\|\boldsymbol{\tau}\|_{T_{\text {div }}^{\prime}}$.

Let

$$
\boldsymbol{\phi}=\frac{-|q \mathbf{I}+\boldsymbol{\tau}|^{r^{\prime} / r-1}(q \mathbf{I}+\boldsymbol{\tau})}{\|q \mathbf{I}+\boldsymbol{\tau}\|_{T^{\prime}}^{r^{\prime}-1}}
$$

Again, $\phi \in T$, and $\|\phi\|_{T}=1$. For this choice of $\phi$,

$$
\begin{aligned}
\frac{[B(\phi),(\boldsymbol{\tau}, q)]}{\|\phi\|_{T}} & =\int_{\Omega} \frac{|q \mathbf{I}+\boldsymbol{\tau}|^{r^{\prime} / r-1}}{\|q \mathbf{I}+\boldsymbol{\tau}\|_{T^{\prime}}^{r^{\prime}-1}}(\boldsymbol{\tau}:(q \mathbf{I}+\boldsymbol{\tau})+q \operatorname{tr}(q \mathbf{I}+\boldsymbol{\tau})) d \Omega \\
& =\int_{\Omega} \frac{|q \mathbf{I}+\boldsymbol{\tau}|^{r^{\prime} / r-1}}{\|q \mathbf{I}+\boldsymbol{\tau}\|_{T^{\prime}}^{r^{\prime}-1}}(q \mathbf{I}+\boldsymbol{\tau}):(q \mathbf{I}+\boldsymbol{\tau}) d \Omega \\
& =\|q \mathbf{I}+\boldsymbol{\tau}\|_{T^{\prime}} \geq\|q \mathbf{I}\|_{T^{\prime}}-\|\boldsymbol{\tau}\|_{T^{\prime}} \\
& =n^{1 / r^{\prime}}\|q\|_{P}-\|\boldsymbol{\tau}\|_{T^{\prime}} \geq\left(n^{1 / r^{\prime}}-1\right)\|q\|_{P} \\
& \geq C\|(\boldsymbol{\tau}, q)\|_{T_{d i v}^{\prime} \times P}
\end{aligned}
$$

\subsection{Inf-sup Condition for $\mathrm{C}$}

The following two lemmas are helpful in establishing the inf-sup condition for $\mathbf{C}$.

Lemma 3.3 Let ${ }_{0} T_{\text {div }}^{\prime}:=\left\{\boldsymbol{\tau} \in T_{\text {div }}^{\prime}: \int_{\Omega} \operatorname{tr}(\boldsymbol{\tau}) d \Omega=0\right\}$. Then, there exists $C>0$ such that for any $\mathbf{u} \in U$

$$
\sup _{\substack{\hat{\boldsymbol{\tau}} \in 0_{d i v}^{\prime} \\ \hat{\boldsymbol{\tau}} \neq 0}} \frac{\int_{\Omega} \mathbf{u} \cdot \operatorname{div} \hat{\boldsymbol{\tau}} d \Omega}{\|\hat{\boldsymbol{\tau}}\|_{T_{d i v}^{\prime}}} \geq C \sup _{\substack{\boldsymbol{\tau} \in T_{d i v}^{\prime} \\ \boldsymbol{\tau} \neq 0}} \frac{\int_{\Omega} \mathbf{u} \cdot \operatorname{div} \boldsymbol{\tau} d \Omega}{\|\boldsymbol{\tau}\|_{T_{d i v}^{\prime}}}
$$

Proof: For $\boldsymbol{\tau} \in T_{d i v}^{\prime}$, let $\left.\boldsymbol{\tau}_{0}=\boldsymbol{\tau}-\frac{1}{n|\Omega|}\left(\int_{\Omega} \operatorname{tr}(\boldsymbol{\tau})\right) d \Omega\right) \mathbf{I}$. Then, $\boldsymbol{\tau}_{0} \in{ }_{0} T_{d i v}^{\prime}$, and div $\boldsymbol{\tau}=\operatorname{div} \boldsymbol{\tau}_{0}$. Let

$$
\boldsymbol{\varsigma}:=\left|\boldsymbol{\tau}_{0}\right|^{r^{\prime} / r-1} \boldsymbol{\tau}_{0}+\frac{\operatorname{sgn}\left(\left(\int_{\Omega} \operatorname{tr}(\boldsymbol{\tau}) d \Omega\right)\left(\int_{\Omega}\left|\boldsymbol{\tau}_{0}\right|^{r^{\prime} / r-1} \boldsymbol{\tau}_{0} d \Omega\right)\right)}{n|\Omega|}\left(\int_{\Omega}\left|\boldsymbol{\tau}_{0}\right|^{r^{\prime} / r-1} \boldsymbol{\tau}_{0} d \Omega\right) \mathbf{I} .
$$


Note that as

$$
\left\|\left|\boldsymbol{\tau}_{0}\right|^{r^{\prime} / r-1} \boldsymbol{\tau}_{0}\right\|_{L^{r}}=\left(\int_{\Omega}\left|\boldsymbol{\tau}_{0}\right|^{r^{\prime}} d \Omega\right)^{1 / r}=\left\|\boldsymbol{\tau}_{0}\right\|_{L^{r^{\prime}}}^{r^{\prime} / r}
$$

and

$$
\left.\left.\left|\int_{\Omega}\right| \boldsymbol{\tau}_{0}\right|^{r^{\prime} / r-1} \operatorname{tr}\left(\boldsymbol{\tau}_{0}\right) d \Omega\left|\leq \sqrt{n} \int_{\Omega}\right| \boldsymbol{\tau}_{0}\right|^{r^{\prime} / r} 1 d \Omega \leq C\left\|\boldsymbol{\tau}_{0}\right\|_{L^{r^{\prime}}}^{r^{\prime} / r}
$$

Thus

$$
\|\boldsymbol{\varsigma}\|_{L^{r}} \leq C\left\|\boldsymbol{\tau}_{0}\right\|_{L^{r^{\prime}}}^{r^{\prime} / r}
$$

We have that

$$
\|\boldsymbol{\tau}\|_{L^{r^{\prime}}}=\sup _{\boldsymbol{\sigma} \in L^{r}} \frac{(\boldsymbol{\tau}, \boldsymbol{\sigma})}{\|\boldsymbol{\sigma}\|_{L^{r}}}
$$

Now, using $\tau_{0} \in{ }_{0} T_{d i v}^{\prime}$

$$
\begin{aligned}
(\boldsymbol{\tau}, \boldsymbol{\varsigma})= & \int_{\Omega}\left|\boldsymbol{\tau}_{0}\right|^{r^{\prime}} d \Omega+\frac{1}{n|\Omega|}\left(\int_{\Omega} \operatorname{tr}(\boldsymbol{\tau}) d \Omega\right)\left(\int_{\Omega}\left|\boldsymbol{\tau}_{0}\right|^{r^{\prime} / r-1} \operatorname{tr}\left(\boldsymbol{\tau}_{0}\right) d \Omega\right) \\
& +\left|\frac{1}{n|\Omega|}\left(\int_{\Omega} \operatorname{tr}(\boldsymbol{\tau}) d \Omega\right)\left(\int_{\Omega}\left|\boldsymbol{\tau}_{0}\right|^{r^{\prime} / r-1} \operatorname{tr}\left(\boldsymbol{\tau}_{0}\right) d \Omega\right)\right| \\
\geq & \left\|\boldsymbol{\tau}_{0}\right\|_{L^{r^{\prime}}}^{r^{\prime}}
\end{aligned}
$$

Therefore, from (3.11), (3.12), and (3.13) we have that $\|\boldsymbol{\tau}\|_{L^{r^{\prime}}} \geq C\left\|\boldsymbol{\tau}_{0}\right\|_{L^{r^{\prime}}}$. Combining the above we obtain

$$
\frac{\int_{\Omega} \mathbf{u} \cdot \operatorname{div} \boldsymbol{\tau} d \Omega}{\|\boldsymbol{\tau}\|_{T_{d i v}^{\prime}}}=\frac{\int_{\Omega} \mathbf{u} \cdot \operatorname{div} \boldsymbol{\tau}_{0} d \Omega}{\|\boldsymbol{\tau}\|_{T_{d i v}^{\prime}}} \leq C \frac{\int_{\Omega} \mathbf{u} \cdot \operatorname{div} \boldsymbol{\tau}_{0} d \Omega}{\left\|\boldsymbol{\tau}_{0}\right\|_{T_{d i v}^{\prime}}}
$$

from which (3.10) then follows.

Lemma 3.4 There exists a constant $c_{2}>0$ such that

$$
\inf _{(\mathbf{u}, \lambda) \in U \times \mathbb{R}} \sup _{(\boldsymbol{\tau}, q) \in T_{d i v}^{\prime} \times P} \frac{[\mathbf{C}(\boldsymbol{\tau}, q),(\mathbf{u}, \lambda)]}{\|(\boldsymbol{\tau}, q)\|_{T_{d i v}^{\prime} \times P}\|(\mathbf{u}, \lambda)\|_{U \times \mathbb{R}}} \geq c_{2} .
$$

Proof: We establish the inf-sup condition by considering two cases.

Case 1.: $|\lambda| \geq\|\mathbf{u}\|_{U}$.

For this case we have

$$
\sup _{(\boldsymbol{\tau}, q) \in T_{d i v}^{\prime} \times P} \frac{[\mathbf{C}(\boldsymbol{\tau}, q),(\mathbf{u}, \lambda)]}{\|(\boldsymbol{\tau}, q)\|_{T_{d i v}^{\prime} \times P}} \geq \frac{[\mathbf{C}(\lambda \mathbf{I}, 0),(\mathbf{u}, \lambda)]}{\|\lambda \mathbf{I}\|_{T_{d i v}^{\prime}}}=\frac{n \lambda^{2}|\Omega|}{|\lambda| n^{r^{\prime} / 2}|\Omega|^{1 / r^{\prime}}} \geq C\|(\mathbf{u}, \lambda)\|_{U \times \mathbb{R}} .
$$

Case 2.: $|\lambda| \leq\|\mathbf{u}\|_{U}$.

Using Lemma 3.3,

$$
\begin{aligned}
\sup _{(\boldsymbol{\tau}, q) \in T_{d i v}^{\prime} \times P} \frac{[\mathbf{C}(\boldsymbol{\tau}, q),(\mathbf{u}, \lambda)]}{\|(\boldsymbol{\tau}, q)\|_{T_{d i v}^{\prime} \times P}} & \geq \sup _{\boldsymbol{\tau}_{0} \in{ }_{0} T_{d i v}^{\prime}} \frac{\left[\mathbf{C}\left(\boldsymbol{\tau}_{0}, 0\right),(\mathbf{u}, \lambda)\right]}{\left\|\boldsymbol{\tau}_{0}\right\|_{T_{d i v}^{\prime}}}=\sup _{\boldsymbol{\tau}_{0} \in{ }_{0} T_{d i v}^{\prime}} \frac{-\int_{\Omega} \mathbf{u} \cdot \operatorname{div} \boldsymbol{\tau}_{0} d \Omega}{\left\|\boldsymbol{\tau}_{0}\right\|_{T_{d i v}^{\prime}}} \\
& \geq C \sup _{\boldsymbol{\tau} \in T_{d i v}^{\prime}} \frac{-\int_{\Omega} \mathbf{u} \cdot \operatorname{div} \boldsymbol{\tau} d \Omega}{\|\boldsymbol{\tau}\|_{T_{d i v}}}
\end{aligned}
$$


Choose $\mathbf{w} \in\left(L^{r^{\prime}}(\Omega)\right)^{n}$ such that $\|\mathbf{u}\|_{L^{r}}\|\mathbf{w}\|_{L^{r^{\prime}}}=\int_{\Omega} \mathbf{u} \cdot \mathbf{w} d \Omega$, and let $\boldsymbol{\tau}$ satisfy div $\boldsymbol{\tau}=\mathbf{w}$ in $\Omega$ with

$$
\|\boldsymbol{\tau}\|_{T_{d i v}^{\prime}} \leq C\|\mathbf{w}\|_{L^{r^{\prime}}}
$$

(see [14] pg. 116). Then,

$$
\begin{aligned}
\sup _{(\boldsymbol{\tau}, q) \in T_{d i v}^{\prime} \times P} \frac{[\mathbf{C}(\boldsymbol{\tau}, q),(\mathbf{u}, \lambda)]}{\|(\boldsymbol{\tau}, q)\|_{T_{d i v}^{\prime} \times P}} & \geq C \frac{-\int_{\Omega} \mathbf{u} \cdot \operatorname{div}(-\boldsymbol{\tau}) d \Omega}{\|-\boldsymbol{\tau}\|_{T_{d i v}^{\prime}}} \geq C \frac{\int_{\Omega} \mathbf{u} \cdot \mathbf{w} d \Omega}{\|\mathbf{w}\|_{L^{r^{\prime}}}} \\
& \geq C\|\mathbf{u}\|_{U} \geq C\|(\mathbf{u}, \lambda)\|_{U \times \mathbb{R}} .
\end{aligned}
$$

We now summarize the above results in the following theorem.

Theorem 3.1 There exists a unique solution $(\boldsymbol{\phi}, \boldsymbol{\psi}, p, \mathbf{u}, \lambda) \in T \times T_{\text {div }}^{\prime} \times P \times U \times \mathbb{R}$ satisfying (2.19)-(2.21). In addition, we have that

$$
\|\phi\|_{T} \leq C\left(\left\|\mathbf{u}_{\Gamma}\right\|_{1-1 / r, r, \Gamma}+\|\mathbf{f}\|_{0, r^{\prime}, \Omega}^{r^{\prime} / r}\right) .
$$

Proof: The bound (3.18) is derived using the inf-sup conditions for $\mathbf{B}$ and $\mathbf{C}$, and (2.2). (See [10] for details.)

\section{Finite Element Approximation}

Let $\Omega \subset \mathbb{R}^{n}$ be a polygonal domain and let $\mathcal{T}_{h}$ be a triangulation of $\Omega$ into triangles $(n=2)$ or tetrahedrals $(n=3)$. Thus

$$
\Omega=\cup K, \quad K \in \mathcal{T}_{h},
$$

and assume that there exist constants $\gamma_{1}, \gamma_{2}$ such that

$$
\gamma_{1} h \leq h_{K} \leq \gamma_{2} \rho_{K}
$$

where $h_{K}$ is the diameter of triangle (tetrahedral) $K, \rho_{K}$ is the diameter of the greatest ball (sphere) included in $K$, and $h=\max _{K \in \mathcal{T}_{h}} h_{K}$. Define the finite-dimensional subspaces $T_{h} \subseteq T, T_{d i v, h}^{\prime} \subseteq T_{d i v}^{\prime}$, $P_{h} \subseteq P$, and $U_{h} \subseteq U$. Then the discrete formulation of (2.13)-(2.15) is defined as:

$$
\begin{aligned}
{\left[\mathbf{A}\left(\phi_{h}\right), \boldsymbol{\varsigma}_{h}\right]+\left[\boldsymbol{\varsigma}_{h}, \mathbf{B}^{*}\left(\boldsymbol{\psi}_{h}, p_{h}\right)\right] } & =0, \forall \boldsymbol{\varsigma}_{h} \in T_{h}, \\
{\left[\mathbf{B}\left(\boldsymbol{\phi}_{h}\right),\left(\boldsymbol{\tau}_{h}, q_{h}\right)\right]+\left[\left(\boldsymbol{\tau}_{h}, q_{h}\right), \mathbf{C}^{*}\left(\mathbf{u}_{h}, \lambda_{h}\right)\right] } & =-\int_{\Gamma}\left(\boldsymbol{\tau}_{h} \cdot \mathbf{n}\right) \cdot \mathbf{u}_{\Gamma} d \Gamma, \\
& \forall\left(\boldsymbol{\tau}_{h}, q_{h}\right) \in T_{d i v, h}^{\prime} \times P_{h}, \\
{\left[\mathbf{C}\left(\boldsymbol{\psi}_{h}, p_{h}\right),\left(\mathbf{v}_{h}, \eta_{h}\right)\right] } & =\int_{\Omega} \mathbf{v}_{h} \cdot \mathbf{f} d \Omega, \forall\left(\mathbf{v}_{h}, \eta_{h}\right) \in U_{h} \times \mathbb{R} .
\end{aligned}
$$

The corresponding discrete kernels of $\mathbf{B}$ and $\mathbf{C}$ are defined similarly. We have

$$
Z_{1 h}:=\left\{\left(\boldsymbol{\tau}_{h}, q_{h}\right) \in T_{d i v, h}^{\prime} \times P_{h}:\left[\mathbf{C}\left(\boldsymbol{\tau}_{h}, q_{h}\right),\left(\mathbf{v}_{h}, \eta_{h}\right)\right]=0, \forall\left(\mathbf{v}_{h}, \eta_{h}\right) \in U_{h} \times \mathbb{R}\right\},
$$

and

$$
Z_{2 h}:=\left\{\boldsymbol{\varsigma}_{h} \in T_{h}:\left[\mathbf{B}\left(\boldsymbol{\varsigma}_{h}\right),\left(\boldsymbol{\tau}_{h}, q_{h}\right)\right]=0, \forall\left(\boldsymbol{\tau}_{h}, q_{h}\right) \in Z_{1 h}\right\}
$$




\subsection{A Priori Estimates}

Theorem 4.1 Let $1<r<2$ and $\mathbf{g}$ satisfy (2.2) and (2.3). Let $(\boldsymbol{\phi}, \boldsymbol{\psi}, p, \mathbf{u}, \lambda) \in T \times T_{\text {div }}^{\prime} \times P \times U \times \mathbb{R}$ solve (2.13)-(2.15). Assume that

(1) There exists a positive constant $c_{1}$ such that

$$
\inf _{\left(\boldsymbol{\tau}_{h}, q_{h}\right) \in Z_{1 h}} \sup _{\boldsymbol{\varsigma}_{h} \in T_{h}} \frac{\left[\mathbf{B}\left(\boldsymbol{\varsigma}_{h}\right),\left(\boldsymbol{\tau}_{h}, q_{h}\right)\right]}{\left\|\boldsymbol{\varsigma}_{h}\right\|_{T}\left\|\left(\boldsymbol{\tau}_{h}, q_{h}\right)\right\|_{T_{d i v}^{\prime} \times P}} \geq c_{1} .
$$

(2) There exists a positive constant $c_{2}$ such that

$$
\inf _{\left(\mathbf{u}_{h}, \lambda_{h}\right) \in U_{h} \times \mathbb{R}} \sup _{\left(\boldsymbol{\tau}_{h}, q_{h}\right) \in T_{d i v, h}^{\prime} \times P_{h}} \frac{\left[\mathbf{C}\left(\boldsymbol{\tau}_{h}, q_{h}\right),\left(\mathbf{u}_{h}, \lambda_{h}\right)\right]}{\left\|\left(\boldsymbol{\tau}_{h}, q_{h}\right)\right\|_{T_{d i v}^{\prime} \times P}\left\|\left(\mathbf{u}_{h}, \lambda_{h}\right)\right\|_{U \times \mathbb{R}}} \geq c_{2} .
$$

Then, for $\mathbf{f} \in\left(L^{r^{\prime}}(\Omega)\right)^{n}$ and $\mathbf{u}_{\Gamma} \in\left(W^{1-1 / r, r}(\Gamma)\right)^{n}$, there exists a unique solution $\left(\phi_{h}, \boldsymbol{\psi}_{h}, p_{h}, \mathbf{u}_{h}, \lambda_{h}\right) \in T_{h} \times T_{d i v, h}^{\prime} \times P_{h} \times U_{h} \times \mathbb{R}$ to the problem (4.2)-(4.4). In addition, we have

$$
\left\|\phi_{h}\right\|_{T} \leq C\left(\left\|\mathbf{u}_{\Gamma}\right\|_{1-1 / r, r, \Gamma}+\|\mathbf{f}\|_{0, r^{\prime}, \Omega}^{r^{\prime} / r}\right)
$$

for some constant $C>0$.

Proof: With the assumptions as stated above, existence and uniqueness of $\left(\phi_{h}, \boldsymbol{\psi}_{h}, p_{h}, \mathbf{u}_{h}, \lambda_{h}\right) \in T_{h} \times T_{d i v, h}^{\prime} \times P_{h} \times U_{h} \times \mathbb{R}$ solving (4.2)-(4.4) follows directly from the continuous solution approach outlined in Section 3 and summarized in Theorem 3.1.

Theorem 4.2 Let

$$
\mathcal{E}\left(\phi, \phi_{h}\right)=\left\|\frac{\left|\phi-\phi_{h}\right|}{|\phi|+\left|\phi_{h}\right|}\right\|_{\infty}^{(2-r) / r} .
$$

Assume the hypotheses of Theorem 4.1 are satisfied. Also assume that for $h$ sufficiently small, there is a constant $c_{3}>0$ such that

$$
\inf _{\left(\boldsymbol{\tau}_{h}, q_{h}\right) \in T_{d i v, h}^{\prime} \times P_{h}\left(\boldsymbol{\varsigma}_{h}, \mathbf{v}_{h}, \eta_{h}\right) \in T_{h} \times U_{h} \times \mathbb{R}} \frac{\left[\mathbf{B}\left(\boldsymbol{\varsigma}_{h}\right),\left(\boldsymbol{\tau}_{h}, q_{h}\right)\right]+\left[\mathbf{C}\left(\boldsymbol{\tau}_{h}, q_{h}\right),\left(\mathbf{v}_{h}, \eta_{h}\right)\right]}{\left\|\left(\boldsymbol{\varsigma}_{h}, \mathbf{v}_{h}, \eta_{h}\right)\right\|_{T \times U \times \mathbb{R}}\left\|\left(\boldsymbol{\tau}_{h}, q_{h}\right)\right\|_{T_{d i v}^{\prime} \times P}^{\prime}} \geq c_{3} .
$$

where $\left\|\left(\boldsymbol{\varsigma}_{h}, \mathbf{v}_{h}, \eta_{h}\right)\right\|_{T \times U \times \mathbb{R}}=\left\|\boldsymbol{\varsigma}_{h}\right\|_{T}+\left\|\mathbf{v}_{h}\right\|_{U}+\left\|\lambda_{h}\right\|_{\mathbb{R}}$. Then

$$
\begin{gathered}
\left\|\boldsymbol{\phi}-\boldsymbol{\phi}_{h}\right\|_{T}^{2}+\int_{\Omega}\left|\mathbf{g}(\boldsymbol{\phi})-\mathbf{g}\left(\boldsymbol{\phi}_{h}\right)\right|\left|\boldsymbol{\phi}-\boldsymbol{\phi}_{h}\right| d \Omega \\
\leq C\left\{\inf _{\boldsymbol{\varsigma}_{h} \in T_{h}}\left(\left\|\boldsymbol{\phi}-\boldsymbol{\varsigma}_{h}\right\|_{T}^{2}+\mathcal{E}\left(\boldsymbol{\phi}, \boldsymbol{\phi}_{h}\right)^{r}\left\|\boldsymbol{\phi}-\boldsymbol{\varsigma}_{h}\right\|_{T}^{r}\right)+\inf _{\mathbf{v}_{h} \in U_{h}}\left\|\mathbf{u}-\mathbf{v}_{h}\right\|_{U}^{2}\right. \\
\left.\quad+\inf _{\boldsymbol{\tau}_{h} \in T_{d i v, h}^{\prime}}\left\|\boldsymbol{\psi}-\boldsymbol{\tau}_{h}\right\|_{T_{d i v}^{\prime}}^{2}+\inf _{q_{h} \in P_{h}}\left\|p-q_{h}\right\|_{P}^{2}\right\}, \\
\left\|\boldsymbol{\psi}-\boldsymbol{\psi}_{h}\right\|_{T_{d i v}^{\prime}}+\left\|p-p_{h}\right\|_{P} \leq C\left\{\inf _{\boldsymbol{\tau}_{h} \in T_{d i v, h}^{\prime}}\left\|\boldsymbol{\psi}-\boldsymbol{\tau}_{h}\right\|_{T_{d i v}^{\prime}}+\inf _{q_{h} \in P_{h}}\left\|p-q_{h}\right\|_{P}\right\} \\
+\mathcal{E}\left(\boldsymbol{\phi}, \boldsymbol{\phi}_{h}\right)\left(\int_{\Omega}\left|\mathbf{g}(\boldsymbol{\phi})-\mathbf{g}\left(\boldsymbol{\phi}_{h}\right)\right|\left|\boldsymbol{\phi}-\boldsymbol{\phi}_{h}\right| d \Omega\right)^{1 / r^{\prime}},
\end{gathered}
$$


and

$$
\left\|\mathbf{u}-\mathbf{u}_{h}\right\|_{U}+\left|\lambda-\lambda_{h}\right| \leq C\left\|\phi-\phi_{h}\right\|_{T}+\inf _{\mathbf{v}_{h} \in U_{h}}\left\|\mathbf{u}-\mathbf{v}_{h}\right\|_{U}
$$

for some constant $C>0$.

Outline of Proof: We briefly describe the how the bounds (4.10)-(4.12) are obtained. Complete details are given in [10].

Using properties (2.2), (2.3), and the definitions

$$
\begin{aligned}
\tilde{Z}_{1 h}:=\left\{\left(\boldsymbol{\tau}_{h}, q_{h}\right) \in T_{d i v, h}^{\prime} \times P_{h}:\left[\mathbf{C}\left(\boldsymbol{\tau}_{h}, q_{h}\right),\left(\mathbf{v}_{h}, \eta_{h}\right)\right]=\int_{\Omega} \mathbf{v}_{h} \cdot \mathbf{f} d \Omega,\right. & \left.\forall\left(\mathbf{v}_{h}, \eta_{h}\right) \in U_{h} \times \mathbb{R}\right\},
\end{aligned}
$$

and

$$
\begin{array}{r}
\tilde{Z}_{2 h}:=\left\{\boldsymbol{\varsigma}_{h} \in T_{h}:\left[\mathbf{B}\left(\boldsymbol{\varsigma}_{h}\right),\left(\boldsymbol{\tau}_{h}, q_{h}\right)\right]+\left[\left(\boldsymbol{\tau}_{h}, q_{h}\right), \mathbf{C}^{*}\left(\mathbf{u}_{h}, \lambda_{h}\right)\right]=-\int_{\Gamma}\left(\boldsymbol{\tau}_{h} \cdot \mathbf{n}\right) \cdot \mathbf{u}_{\Gamma} d \Gamma\right. \\
\left.\forall\left(\boldsymbol{\tau}_{h}, q_{h}\right) \in \tilde{Z}_{1 h}\right\},
\end{array}
$$

we obtain the estimate

$$
\begin{aligned}
\left\|\boldsymbol{\phi}-\boldsymbol{\phi}_{h}\right\|_{T}^{2}+\int_{\Omega}\left|\mathbf{g}(\boldsymbol{\phi})-\mathbf{g}\left(\boldsymbol{\phi}_{h}\right)\right|\left|\boldsymbol{\phi}-\boldsymbol{\phi}_{h}\right| d \Omega \leq & C\left\{\inf _{\boldsymbol{\varsigma}_{h} \in \tilde{Z}_{2 h}}\left(\left\|\boldsymbol{\phi}-\boldsymbol{\varsigma}_{h}\right\|_{T}^{2}+\mathcal{E}\left(\boldsymbol{\phi}, \boldsymbol{\phi}_{h}\right)^{r}\left\|\boldsymbol{\phi}-\boldsymbol{\varsigma}_{h}\right\|_{T}^{r}\right)\right. \\
& \left.+\inf _{\left(\boldsymbol{\tau}_{h}, q_{h}\right) \in \tilde{Z}_{1 h}}\left(\left\|\boldsymbol{\psi}-\boldsymbol{\tau}_{h}\right\|_{T^{\prime}}^{2}+\left\|p-q_{h}\right\|_{P}^{2}\right)\right\} .
\end{aligned}
$$

A "lifting argument" which requires the combined inf-sup condition (4.9) is then used to lift the "best approximations" in $\tilde{Z}_{1 h}$ and $\tilde{Z}_{2 h}$ to the "best approximations" in $T_{h}, U_{h}, T_{d i v, h}^{\prime}$, and $P_{h}$. The bounds (4.10)-(4.12) are then obtained using the discrete inf-sup conditions (4.5) and (4.6).

Remark 4.1 As previously noted, $\mathcal{E}\left(\phi, \phi_{h}\right) \leq 1$. In addition, if $1 /\left(|\phi|+\left|\phi_{h}\right|\right) \leq C$ for some constant $C>0$, then

$$
\mathcal{E}\left(\phi, \phi_{h}\right) \leq \min \left\{1, C\left\|\phi-\phi_{h}\right\|_{\infty}^{(2-r) / r}\right\}
$$

Furthermore, if $\left\|\phi-\phi_{h}\right\|_{\infty} \sim\left\|\phi-\phi_{h}\right\|_{T}$, the estimates (4.10)-(4.12) may be written as

$$
\begin{aligned}
\left\|\boldsymbol{\phi}-\boldsymbol{\phi}_{h}\right\|_{T}+\left\|\boldsymbol{\psi}-\boldsymbol{\psi}_{h}\right\|_{T_{d i v}^{\prime}} & +\left\|p-p_{h}\right\|_{P}+\left\|\mathbf{u}-\mathbf{u}_{h}\right\|_{U}+\left|\lambda-\lambda_{h}\right| \\
\leq C\left\{\inf _{\boldsymbol{\varsigma}_{h} \in T_{h}}\left\|\boldsymbol{\phi}-\boldsymbol{\varsigma}_{h}\right\|_{T}+\inf _{\mathbf{v}_{h} \in U_{h}}\left\|\mathbf{u}-\mathbf{v}_{h}\right\|_{U}\right. & \\
& \left.+\inf _{\boldsymbol{\tau}_{h} \in T_{d i v, h}^{\prime}}\left\|\boldsymbol{\psi}-\boldsymbol{\tau}_{h}\right\|_{T_{d i v}^{\prime}}+\inf _{q_{h} \in P_{h}}\left\|p-q_{h}\right\|_{P}\right\} .
\end{aligned}
$$




\section{2 $T_{d i v}^{\prime}$ Approximation Using Raviart-Thomas Elements}

In this section, we show that approximating spaces of discontinuous piecewise polynomials and Raviart-Thomas elements are suitable for problem (2.13)-(2.15). Specifically, we show that these spaces satisfy the inf-sup conditions (4.5), (4.6), and (4.9).

Let $n=2$. Let $K \in \mathcal{T}_{h}$ and let $\mathbb{P}_{k}(K)$ be the set of all polynomials in the variables $x_{1}, x_{2}$ of degree less than or equal to $k$ defined on the triangle $K$. Let $\mathbb{R T}_{k}(K)$ be the 2-vector of Raviart-Thomas elements [25, 27] on $K$ defined by

$$
\mathbb{R} \mathbb{T}_{k}(K)=\left(\mathbb{P}_{k}(K)\right)^{2}+\left[\begin{array}{l}
x_{1} \\
x_{2}
\end{array}\right] \mathbb{P}_{k}(K)
$$

For $k \geq 0$, define the following discrete spaces:

$$
\begin{aligned}
& T_{h}:=\left\{\boldsymbol{\phi} \in T:\left.\phi\right|_{K} \in\left(\mathbb{P}_{k}(K)\right)^{2 \times 2}, \quad \forall K \in \mathcal{T}_{h}\right\}, \\
& T_{d i v, h}^{\prime}:=\left\{\boldsymbol{\psi} \in T_{\text {div }}^{\prime}: \boldsymbol{\psi}=\left.\left(\begin{array}{ll}
\boldsymbol{\psi}_{1} & \boldsymbol{\psi}_{2}
\end{array}\right)^{\mathrm{T}}\right|_{K} \in\left(\mathbb{R T}_{k}(K)\right)^{2},\right. \\
&\left.\left.\left(\psi_{i 1} \quad \psi_{i 2}\right)^{\mathrm{T}}\right|_{K} \in \mathbb{R} \mathbb{T}_{k}(K), \quad \forall i \in\{1,2\}, \quad \forall K \in \mathcal{T}_{h}\right\}, \\
& P_{h}:=\left\{p \in P:\left.p\right|_{K} \in \mathbb{P}_{k}(K), \quad \forall K \in \mathcal{T}_{h}\right\} \\
& U_{h}:=\left\{\mathbf{u} \in U:\left.\mathbf{u}\right|_{K} \in\left(\mathbb{P}_{k}(K)\right)^{2}, \quad \forall K \in \mathcal{T}_{h}\right\} .
\end{aligned}
$$

Remark 4.2 There is no interelement continuity requirement on the spaces $T_{h}, U_{h}$, and $P_{h}$.

Let $s>1$ and let $\mathcal{I}_{h}^{k}:\left(W^{1, s}(\Omega)\right)^{2 \times 2} \longrightarrow T_{d i v, h}^{\prime}$ be the $k$-th order Raviart-Thomas interpolation operator $[25,7,9]$, defined by, for row $j=1,2$ of $\boldsymbol{\tau} \in T_{\text {div }}^{\prime}$,

$$
\begin{aligned}
\int_{e_{i}}\left(\boldsymbol{\tau}_{j}-\mathcal{I}_{h}^{k} \boldsymbol{\tau}_{j}\right) \cdot \mathbf{n}_{e_{i}} v_{k} d s & =0, \quad \forall v_{k} \in \mathbb{P}_{k}(K), \quad \forall e_{i} \in \partial K, \quad i=1,2,3, \quad \forall K \in \mathcal{T}_{h}, \\
\int_{K}\left(\boldsymbol{\tau}_{j}-\mathcal{I}_{h}^{k} \boldsymbol{\tau}_{j}\right) \cdot \mathbf{v}_{k-1} d K & =0, \quad \forall \mathbf{v}_{k-1} \in\left(\mathbb{P}_{k-1}(K)\right)^{2}, \quad \forall K \in \mathcal{T}_{h},
\end{aligned}
$$

where $\mathbf{n}_{e_{i}}$ denotes the outer unit normal vector to edge $e_{i}$ of $K$. Then, for $0 \leq m \leq k+1$, we have

$$
\begin{gathered}
\left\|\boldsymbol{\tau}-\mathcal{I}_{h}^{k} \boldsymbol{\tau}\right\|_{0, r^{\prime}, \Omega} \leq C h^{m}|\boldsymbol{\tau}|_{m, r^{\prime}, \Omega}, \\
\left\|\operatorname{div}\left(\boldsymbol{\tau}-\mathcal{I}_{h}^{k} \boldsymbol{\tau}\right)\right\|_{0, r^{\prime}, \Omega} \leq C h^{m}|\operatorname{div} \boldsymbol{\tau}|_{m, r^{\prime}, \Omega},
\end{gathered}
$$

and, for $\mathbf{v} \in U$,

$$
\int_{\Omega} \mathbf{v} \cdot \operatorname{div}\left(\boldsymbol{\tau}-\mathcal{I}_{h}^{k} \boldsymbol{\tau}\right) d \Omega=0, \quad \forall \boldsymbol{\tau} \in T_{d i v}^{\prime}
$$

In the lowest-order case, i.e., $k=0$, for $\left(\boldsymbol{\tau}_{h}, q_{h}\right) \in Z_{1 h}$,

$$
\boldsymbol{\phi}^{*}=\frac{-\left|q_{h} \mathbf{I}+\boldsymbol{\tau}_{h}\right|^{r^{\prime} / r-1}\left(q_{h} \mathbf{I}+\boldsymbol{\tau}_{h}\right)}{\left\|q_{h} \mathbf{I}+\boldsymbol{\tau}_{h}\right\|_{T^{\prime}}^{r^{\prime}-1}} \in T_{h} .
$$


The proof of the discrete inf-sup condition for $\mathbf{B}$ then follows as in the continuous case. However, for higher-order approximations, $\boldsymbol{\phi}^{*}$ defined by (4.20) for $\left(\boldsymbol{\tau}_{h}, q_{h}\right) \in Z_{1 h}$ is not a polynomial and hence not in $T_{h}$. In these cases a suitable projection of $\phi^{*}$ is required.

From [1], if $f \in L^{2}(\Omega)$, then $f \in L^{r}(\Omega)$ for $1<r<2$, and $\|f\|_{0, r, \Omega} \leq|\Omega|^{\frac{2-r}{2 r}}\|f\|_{0,2, \Omega}$. Also the following relationship of norms in $L^{2}(\Omega)$ and $L^{r}(\Omega)$ holds.

Lemma 4.1 Let $f, g \in L^{2}(\Omega)$ and $1<r<2$. If $\|f\|_{0, r}=\mu$ and $\|f\|_{0,2}=\|g\|_{0,2}$, then $\|g\|_{0, r} \leq \mu$.

Proof: Assume $\|f\|_{0, r}=\mu$ and $\|f\|_{0,2}=\|g\|_{0,2}$. Using Hölder's inequality we have

$$
\|f\|_{0,2}^{2}=\int_{\Omega}|f|^{2} d \Omega \leq\left(\int_{\Omega}|f|^{r} d \Omega\right)^{2 / r}\left(\int_{\Omega} 1 d \Omega\right)^{(r-2) / r} \leq \mu^{2}|\Omega|^{(r-2) / r},
$$

thus

$$
\|f\|_{0,2} \leq \mu|\Omega|^{(r-2) / 2 r}
$$

Also,

$$
\|g\|_{0, r}^{r}=\int_{\Omega}|g|^{r} d \Omega \leq\|g\|_{0,2}^{r}|\Omega|^{(2-r) / 2}=\|f\|_{0,2}^{r}|\Omega|^{(2-r) / 2} \leq \mu^{r}|\Omega|^{(r-2+2-r) / r}=\mu^{r} .
$$

Lemma 4.2 For the choices of $T_{h}, T_{d i v, h}^{\prime}, P_{h}$, and $U_{h}$ above, there exists a positive constant $c_{1}$ such that

$$
\inf _{\left(\boldsymbol{\tau}_{h}, q_{h}\right) \in Z_{1 h}} \sup _{\boldsymbol{\phi}_{h} \in T_{h}} \frac{\left[\mathbf{B}\left(\phi_{h}\right),\left(\boldsymbol{\tau}_{h}, q_{h}\right)\right]}{\left\|\phi_{h}\right\|_{T}\left\|\left(\boldsymbol{\tau}_{h}, q_{h}\right)\right\|_{T_{d i v}^{\prime} \times P}} \geq c_{1} .
$$

Proof: Note that for $\left(\phi_{h}, q_{h}\right) \in Z_{1 h}$, div $\boldsymbol{\tau}_{h}=\mathbf{0}$ implies $\left.\boldsymbol{\tau}_{h}\right|_{K} \in\left(\mathbb{P}_{k}(K)\right)^{2 \times 2}$ for all $K \in \mathcal{T}_{h}$. We also have that $\left.\left(\boldsymbol{\tau}_{h}+q_{h} \mathbf{I}\right)\right|_{K} \in\left(\mathbb{P}_{k}(K)\right)^{2 \times 2}$ for all $K \in \mathcal{T}_{h}$. Thus $\left(\boldsymbol{\tau}_{h}, q_{h}\right) \in Z_{1 h}$ implies $\boldsymbol{\tau}_{h} \in T_{h}$ and $\left(\boldsymbol{\tau}_{h}+q_{h} \mathbf{I}\right) \in T_{h}$.

Assume that $\left\|q_{h}\right\|_{P} \leq\left\|\boldsymbol{\tau}_{h}\right\|_{T_{d i v}^{\prime}}$. Let $\boldsymbol{\tau}_{h}^{0}=\boldsymbol{\tau}_{h}-\frac{1}{n} \operatorname{tr}\left(\boldsymbol{\tau}_{h}\right) \mathbf{I}$, and

$$
\phi^{*}=-\left|\boldsymbol{\tau}_{h}^{0}\right|^{r^{\prime} / r-1} \boldsymbol{\tau}_{h}^{0} /\left\|\boldsymbol{\tau}_{h}^{0}\right\|_{T^{\prime}}^{r^{\prime}-1}
$$

Then $\left\|\phi^{*}\right\|_{T}=1$ and

$$
\left(\phi^{*}, \phi_{h}\right)=\int_{\Omega} \phi^{*}: \phi_{h} d \Omega, \quad \forall \phi_{h} \in T_{h}
$$

defines a continuous linear functional on $T_{h}$. Note that $T_{h}$ equipped with the $L^{2}$ inner product is a Hilbert space. Then by the Riesz Representation Theorem there exists a $\varsigma_{h} \in T_{h}$ such that

$$
\left(\varsigma_{h}, \phi_{h}\right)=\int_{\Omega} \varsigma_{h}: \phi_{h} d \Omega=\int_{\Omega} \phi^{*}: \phi_{h} d \Omega=\left(\phi^{*}, \phi_{h}\right), \quad \forall \phi_{h} \in T_{h},
$$

with

$$
\left\|\varsigma_{h}\right\|_{0,2}=\left\|\phi^{*}\right\|_{0,2} \cdot
$$


From Lemma 4.1, we have that $\left\|\boldsymbol{\varsigma}_{h}\right\|_{T} \leq 1$. Then $\left[\mathbf{B}\left(\boldsymbol{\varsigma}_{h}\right),\left(\boldsymbol{\tau}_{h}, p_{h}\right)\right]=\left[\mathbf{B}\left(\boldsymbol{\phi}^{*}\right),\left(\boldsymbol{\tau}_{h}, p_{h}\right)\right]$ for all $\left(\boldsymbol{\tau}_{h}, q_{h}\right) \in Z_{1 h}$. Continuing as in (3.7), the result is shown as in Case 1 of Lemma 3.2.

Now assume $\left\|q_{h}\right\|_{P} \geq\left\|\boldsymbol{\tau}_{h}\right\|_{T_{\text {div }}^{\prime}}$. Let

$$
\boldsymbol{\phi}^{*}=\frac{-\left|q_{h} \mathbf{I}+\boldsymbol{\tau}_{h}\right|^{r^{\prime} / r-1}\left(q_{h} \mathbf{I}+\boldsymbol{\tau}_{h}\right)}{\left\|q_{h} \mathbf{I}+\boldsymbol{\tau}_{h}\right\|_{T^{\prime}}^{r^{\prime}-1}} .
$$

Again let $\varsigma_{h} \in T_{h}$ satisfy $\left(\varsigma_{h}, \phi_{h}\right)=\left(\phi^{*}, \phi_{h}\right)$ for all $\phi_{h} \in T_{h}$ and $\left\|\phi^{*}\right\|_{0,2}=\left\|\varsigma_{h}\right\|_{0,2} \leq\left\|\phi^{*}\right\|_{T}=1$. Continuing as in the proof of Case 2 of Lemma 3.2, the result is shown.

Lemma 4.3 For the choices of $T_{h}, T_{d i v, h}^{\prime}, P_{h}$, and $U_{h}$ above, there exists a positive constant $c_{2}$ such that

$$
\inf _{\left(\mathbf{u}_{h}, \lambda_{h}\right) \in U_{h} \times \mathbb{R}} \sup _{\left(\boldsymbol{\tau}_{h}, q_{h}\right) \in T_{d i v, h}^{\prime} \times P_{h}} \frac{\left[\mathbf{C}\left(\boldsymbol{\tau}_{h}, q_{h}\right),\left(\mathbf{u}_{h}, \lambda_{h}\right)\right]}{\left\|\left(\boldsymbol{\tau}_{h}, q_{h}\right)\right\|_{T_{d i v}^{\prime} \times P}\left\|\left(\mathbf{u}_{h}, \lambda_{h}\right)\right\|_{U \times \mathbb{R}}} \geq c_{2} .
$$

Proof: As in the approach to the proof of Lemma 3.4, we consider two cases:

Case 1: $\left|\lambda_{h}\right| \geq\left\|\mathbf{u}_{h}\right\|_{U}$.

The choice $\left(\boldsymbol{\tau}_{h}, q_{h}\right)=\left(\lambda_{h} \mathbf{I}, 0\right) \in T_{d i v, h}^{\prime} \times P_{h}$ shows the result as in Case 1 of the proof of Lemma 3.4 .

Case 2: $\left|\lambda_{h}\right| \leq\left\|\mathbf{u}_{h}\right\|_{U}$.

Note that Lemma 3.3 applies to the subspace $T_{d i v, h}^{\prime} \subset T_{d i v}^{\prime}$, thus we have

$$
\begin{aligned}
\sup _{\left(\boldsymbol{\tau}_{h}, q_{h}\right) \in T_{d i v, h}^{\prime} \times P_{h}} \frac{\left[\mathbf{C}\left(\boldsymbol{\tau}_{h}, q_{h}\right),\left(\mathbf{u}_{h}, \lambda_{h}\right)\right]}{\left\|\left(\boldsymbol{\tau}_{h}, q_{h}\right)\right\|_{T_{d i v}^{\prime} \times P}^{\prime}} & \geq \sup _{\boldsymbol{\tau}_{0} \in{ }_{0} T_{d i v, h}^{\prime}} \frac{\left[\mathbf{C}\left(\boldsymbol{\tau}_{0}, 0\right),\left(\mathbf{u}_{h}, \lambda_{h}\right)\right]}{\left\|\boldsymbol{\tau}_{0}\right\|_{T_{d i v}^{\prime}}} \\
& =\sup _{\boldsymbol{\tau}_{0} \in T_{0}^{\prime} T_{d i v, h}^{\prime}} \frac{-\int_{\Omega} \mathbf{u}_{h} \cdot \operatorname{div} \boldsymbol{\tau}_{0} d \Omega}{\left\|\boldsymbol{\tau}_{0}\right\|_{T_{d i v, h}^{\prime}}} \\
& \geq C \sup _{\boldsymbol{\tau}_{\in \in T_{d i v, h}^{\prime}}} \frac{-\int_{\Omega} \mathbf{u}_{h} \cdot \operatorname{div} \boldsymbol{\tau}_{h} d \Omega}{\left\|\boldsymbol{\tau}_{h}\right\|_{T_{d i v, h}^{\prime}}}
\end{aligned}
$$

Now we proceed in a manner similar to that of Proposition 5 of [23] (as well as Proposition 3.1 of [13]). Let $\mathbf{w}$ be the solution of the Laplacian problem

$$
\begin{aligned}
-\Delta \mathbf{w} & =\left|\mathbf{u}_{h}\right|^{r-2} \mathbf{u}_{h}, & & \text { in } \Omega, \\
\mathbf{w} & =\mathbf{0}, & & \text { on } \Gamma .
\end{aligned}
$$

Note that $\left|\mathbf{u}_{h}\right|^{r-2} \mathbf{u}_{h} \in\left(W^{0, r^{\prime}}(\Omega)\right)^{2}$. Hence, from [17], this problem has a unique solution $\mathbf{w} \in$ $\left(W_{0}^{2, r}(\Omega)\right)^{2}$, and there exists a constant $C>0$ such that

$$
\|\mathbf{w}\|_{2, r^{\prime}, \Omega} \leq C\left\|\left|\mathbf{u}_{h}\right|^{r-2} \mathbf{u}_{h}\right\|_{0, r^{\prime}, \Omega}=C\left(\int_{\Omega}\left|\mathbf{u}_{h}\right|^{r^{\prime}(r-1)} d \Omega\right)^{(r-1) / r}=C\left\|\mathbf{u}_{h}\right\|_{0, r, \Omega}^{r-1} .
$$

Now let $\boldsymbol{\tau}^{*}=\nabla \mathbf{w}$. Thus from (4.24) we have

$$
\left\|\boldsymbol{\tau}^{*}\right\|_{1, r^{\prime}, \Omega}=\|\mathbf{w}\|_{2, r^{\prime}, \Omega} \leq C\left\|\mathbf{u}_{h}\right\|_{0, r, \Omega}^{r-1},
$$


and $\nabla \cdot \boldsymbol{\tau}^{*}=\Delta \mathbf{w}=-\left|\mathbf{u}_{h}\right|^{r-2} \mathbf{u}_{h}$. Thus $\boldsymbol{\tau}^{*} \in T_{\text {div }}^{\prime}$ and we have

$$
\left\|\boldsymbol{\tau}^{*}\right\|_{T_{d i v}^{\prime}} \leq C\left\|\mathbf{u}_{h}\right\|_{0, r, \Omega}^{r-1}
$$

Let $\boldsymbol{\tau}_{h}=\mathcal{I}_{h}^{k} \tau^{*}$. Then we have, from (4.19), that

$$
-\int_{\Omega} \mathbf{u}_{h} \cdot \operatorname{div} \boldsymbol{\tau}_{h} d \Omega=-\int_{\Omega} \mathbf{u}_{h} \cdot \operatorname{div} \boldsymbol{\tau}^{*} d \Omega=\int_{\Omega}\left|\mathbf{u}_{h}\right|^{r-2} \mathbf{u}_{h} \cdot \mathbf{u}_{h} d \Omega=\left\|\mathbf{u}_{h}\right\|_{U}^{r} .
$$

The estimate (4.18) and the triangle inequality gives, for $m=0$,

$$
\left\|\nabla \cdot \tau_{h}\right\|_{0, r^{\prime}, \Omega} \leq C\left\|\nabla \cdot \tau^{*}\right\|_{0, r^{\prime}, \Omega} .
$$

Then, from (4.17), (4.26), (4.28), and the triangle inequality, we have

$$
\begin{aligned}
\left\|\boldsymbol{\tau}_{h}\right\|_{T_{d i v}^{\prime}} & \leq\left\|\boldsymbol{\tau}^{h}\right\|_{0, r^{\prime}, \Omega}+\left\|\nabla \cdot \boldsymbol{\tau}_{h}\right\|_{0, r^{\prime}, \Omega} \\
& \leq C\left(\left\|\boldsymbol{\tau}^{*}\right\|_{0, r^{\prime}, \Omega}+\left\|\boldsymbol{\tau}^{*}-\boldsymbol{\tau}_{h}\right\|_{0, r^{\prime}, \Omega}+\left\|\nabla \cdot \boldsymbol{\tau}^{*}\right\|_{0, r^{\prime}, \Omega}\right) \\
& \leq C\left(\left\|\boldsymbol{\tau}^{*}\right\|_{T_{d i v}^{\prime}}+h\left\|\boldsymbol{\tau}^{*}\right\|_{1, r^{\prime}, \Omega}+\|\mathbf{u}\|_{0, r, \Omega}^{r-1}\right) \\
& \leq C\left(\left\|\mathbf{u}_{h}\right\|_{0, r, \Omega}^{r-1}+h\left\|\mathbf{u}_{h}\right\|_{0, r, \Omega}^{r-1}+\left\|\mathbf{u}_{h}\right\|_{0, r, \Omega}^{r-1}\right) \leq C\left\|\mathbf{u}_{h}\right\|_{U}^{r-1}
\end{aligned}
$$

Combining (4.23), (4.27), and (4.29), we have that

$$
\begin{gathered}
\sup _{\left(\boldsymbol{\tau}_{h}, q_{h}\right) \in T_{d i v, h}^{\prime} \times P_{h}} \frac{\left[\mathbf{C}\left(\boldsymbol{\tau}_{h}, q_{h}\right),\left(\mathbf{u}_{h}, \lambda_{h}\right)\right]}{\left\|\left(\boldsymbol{\tau}_{h}, q_{h}\right)\right\|_{T_{d i v}^{\prime} \times P}} \geq \frac{-\int_{\Omega} \mathbf{u}_{h} \cdot \operatorname{div} \boldsymbol{\tau}_{h} d \Omega}{\left\|\boldsymbol{\tau}_{h}\right\|_{T_{d i v}^{\prime}}} \geq \frac{\left\|\mathbf{u}_{h}\right\|_{U}^{r}}{C\left\|\mathbf{u}_{h}\right\|_{U}^{r-1}} \\
\geq C\left\|\mathbf{u}_{h}\right\|_{U} \geq c_{2}\left\|\left(\mathbf{u}_{h}, \lambda_{h}\right)\right\|_{U \times \mathbb{R}}
\end{gathered}
$$

and thus we obtain (4.22).

Before showing the inf-sup condition (4.9) holds for the chosen approximation spaces, we first discuss some properties of the Raviart-Thomas elements. Let $K \in \mathcal{T}_{h}$ and let $\mathbf{r} \in \mathbb{R} \mathbb{T}_{k}(K)$. Then $\mathbf{r}$ can be written as $\mathbf{r}=\mathbf{r}^{k}+\mathbf{r}^{*}$, where $\mathbf{r}^{k} \in\left(\mathbb{P}_{k}(K)\right)^{2}$ and the components of $\mathbf{r}^{*}$ consist of polynomial terms of degree $k+1$ only. In fact, $\mathbf{r}^{*}$ can be written as

$$
\mathbf{r}^{*}=\left[\begin{array}{l}
x_{1} \\
x_{2}
\end{array}\right] \sum_{j=0}^{k} \gamma_{j} x_{1}^{k-j} x_{2}^{j}=\left[\begin{array}{l}
\sum_{j=0}^{k} \gamma_{j} x_{1}^{k-j+1} x_{2}^{j} \\
\sum_{j=0}^{k} \gamma_{j} x_{1}^{k-j} x_{2}^{j+1}
\end{array}\right]
$$

for some constants $\gamma_{j}, j=0, \ldots, k$.

We can also write $\operatorname{div} \mathbf{r}=\operatorname{div} \mathbf{r}^{k}+\operatorname{div} \mathbf{r}^{*}$, where $\operatorname{div} \mathbf{r}^{k}$ is a polynomial of degree at most $k-1$ and $\operatorname{div} \mathbf{r}^{*}$ is a polynomial with terms of degree $k$ only. It is important to note that if $\operatorname{div} \mathbf{r}=0$, then $\operatorname{div} \mathbf{r}^{*}=0$. For Raviart-Thomas elements we have that the norm of the gradient of the highest-degree terms can be bounded by the norm of the divergence. 
Lemma 4.4 Let $K \in \mathcal{T}_{h}$ and let $\mathbf{r}=\mathbf{r}^{k}+\mathbf{r}^{*} \in \mathbb{R T}_{k}(K)$ where the components of $\mathbf{r}^{*}$ consist of polynomial terms of degree $k+1$ only. Then there exists a constant $\tilde{C}>0$, independent of $K$, such that

$$
\left\|\nabla \mathbf{r}^{*}\right\|_{0, r^{\prime}, K} \leq \tilde{C}\|\operatorname{div} \mathbf{r}\|_{0, r^{\prime}, K}
$$

Outline of Proof: The proof follows from the special form of the Raviart-Thomas element and the equivalence of finite dimensional norms. (See [10] for complete details.)

The above result can be applied to the tensor space $T_{d i v, h}^{\prime}$ to obtain, for $\boldsymbol{\tau}_{h}=\boldsymbol{\tau}^{k}+\boldsymbol{\tau}^{*}$ where the components of $\boldsymbol{\tau}^{*}$ consist of polynomial terms of degree $k+1$ only,

$$
\left\|\nabla \boldsymbol{\tau}^{*}\right\|_{0, r^{\prime}, K} \leq \tilde{C}\left\|\operatorname{div} \boldsymbol{\tau}_{h}\right\|_{0, r^{\prime}, K}, \quad \forall K \in \mathcal{T}_{h} .
$$

Let $\Pi_{k}: T_{d i v, h}^{\prime} \longrightarrow T_{h}$ be the classical Lagrangian $\mathcal{P}_{k}$ interpolation operator $([9])$ and define

$$
\hat{\tau}=\boldsymbol{\tau}^{k}+\Pi_{k} \boldsymbol{\tau}^{*}
$$

Note that $\left.\hat{\boldsymbol{\tau}}\right|_{K} \in\left(\mathbb{P}_{k}(K)\right)^{2 \times 2}$ for all $K \in \mathcal{T}_{h}$, and $\operatorname{div} \boldsymbol{\tau}_{h}=\mathbf{0}$ implies $\boldsymbol{\tau}^{*}=\mathbf{0}$ and $\hat{\boldsymbol{\tau}}=\boldsymbol{\tau}_{h}$. Then, using (4.31) and standard polynomial approximation properties [6,9], the error associated in the approximation of $\boldsymbol{\tau}_{h}$ by $\hat{\boldsymbol{\tau}}$ is given by

$$
\begin{aligned}
& \left\|\boldsymbol{\tau}_{h}-\hat{\boldsymbol{\tau}}\right\|_{0, r^{\prime}, \Omega}=\left\|\boldsymbol{\tau}^{*}-\Pi_{k} \boldsymbol{\tau}^{*}\right\|_{0, r^{\prime}, \Omega} \leq C h\left(\sum_{K \in \mathcal{T}_{h}}\left\|\nabla \boldsymbol{\tau}^{*}\right\|_{0, r^{\prime}, K}^{r^{\prime}}\right)^{1 / r^{\prime}} \\
& \leq C h\left(\sum_{K \in \mathcal{T}_{h}} \tilde{C}\left\|\operatorname{div} \boldsymbol{\tau}_{h}\right\|_{0, r^{\prime}, K}^{r^{\prime}}\right)^{1 / r^{\prime}} \leq C \tilde{C} h\left\|\operatorname{div} \boldsymbol{\tau}_{h}\right\|_{0, r^{\prime}, \Omega}=\hat{C} h\left\|\operatorname{div} \boldsymbol{\tau}_{h}\right\|_{0, r^{\prime}, \Omega} .
\end{aligned}
$$

Lemma 4.5 For $h$ sufficiently small, there is a constant $c_{3}>0$ such that

$$
\inf _{\left(\boldsymbol{\tau}_{h}, q_{h}\right) \in T_{d i v, h}^{\prime} \times P_{h}} \sup _{\left(\boldsymbol{\varsigma}_{h}, \mathbf{v}_{h}, \eta_{h}\right) \in T_{h} \times U_{h} \times \mathbb{R}} \frac{\left[\mathbf{B}\left(\boldsymbol{\varsigma}_{h}\right),\left(\boldsymbol{\tau}_{h}, q_{h}\right)\right]+\left[\mathbf{C}\left(\boldsymbol{\tau}_{h}, q_{h}\right),\left(\mathbf{v}_{h}, \eta_{h}\right)\right]}{\left\|\left(\boldsymbol{\varsigma}_{h}, \mathbf{v}_{h}, \eta_{h}\right)\right\|_{T \times U \times \mathbb{R}}\left\|\left(\boldsymbol{\tau}_{h}, q_{h}\right)\right\|_{T_{d i v}^{\prime} \times P}} \geq c_{3} .
$$

where $\left\|\left(\boldsymbol{\varsigma}_{h}, \mathbf{v}_{h}, \eta_{h}\right)\right\|_{T \times U \times \mathbb{R}}=\left\|\boldsymbol{\varsigma}_{h}\right\|_{T}+\left\|\mathbf{v}_{h}\right\|_{U}+\left\|\lambda_{h}\right\|_{\mathbb{R}}$.

Proof: Again we need to consider the two cases $\left\|\boldsymbol{\tau}_{h}\right\|_{T_{d i v}^{\prime}} \leq\left\|q_{h}\right\|_{P}$ and $\left\|\boldsymbol{\tau}_{h}\right\|_{T_{d i v}^{\prime}}>\left\|q_{h}\right\|_{P}$.

Case 1: $\left(\left\|\boldsymbol{\tau}_{h}\right\|_{T_{\text {div }}^{\prime}} \leq\left\|q_{h}\right\|_{P}\right)$

Let $\hat{\boldsymbol{\tau}}$ be as defined in (4.32). Let

$$
\phi^{*}=\frac{-1}{\left\|q_{h} \mathbf{I}+\hat{\boldsymbol{\tau}}\right\|_{T^{\prime}}^{r^{\prime}-1}}\left|q_{h} \mathbf{I}+\hat{\boldsymbol{\tau}}\right|^{r^{\prime} / r-1}\left(q_{h} \mathbf{I}+\hat{\boldsymbol{\tau}}\right) .
$$

Note that $\left\|\phi^{*}\right\|_{T}=1$. Now, there exists a $\varsigma_{h} \in T_{h}$ such that

$$
\int_{\Omega} \varsigma_{h}: \phi_{h} d \Omega=\int_{\Omega} \phi^{*}: \phi_{h} d \Omega, \quad \forall \phi_{h} \in T_{h}
$$


with $\left\|\boldsymbol{\varsigma}_{h}\right\|_{T} \leq\left\|\phi^{*}\right\|_{T}=1$. Then, since $q_{h} \mathbf{I}+\hat{\boldsymbol{\tau}} \in T_{h}$, we have that

$$
\int_{\Omega} \boldsymbol{\varsigma}_{h}:\left(q_{h} \mathbf{I}+\hat{\boldsymbol{\tau}}\right) d \Omega=\int_{\Omega} \boldsymbol{\phi}^{*}:\left(q_{h} \mathbf{I}+\hat{\boldsymbol{\tau}}\right) d \Omega .
$$

Let $\mathbf{v}_{h}=\mathbf{0}$ and $\eta_{h}=0$. Then we have

$$
\begin{aligned}
\frac{\left[\mathbf{B}\left(\boldsymbol{\varsigma}_{h}\right),\left(\boldsymbol{\tau}_{h}, q_{h}\right)\right]}{\left\|\boldsymbol{\varsigma}_{h}\right\|_{T}} & =-\int_{\Omega} \boldsymbol{\varsigma}_{h}:\left(q_{h} \mathbf{I}+\boldsymbol{\tau}_{h}\right) d \Omega \\
& =-\int_{\Omega} \boldsymbol{\varsigma}_{h}:\left(q_{h} \mathbf{I}+\hat{\boldsymbol{\tau}}\right) d \Omega-\int_{\Omega} \boldsymbol{\varsigma}_{h}:\left(\boldsymbol{\tau}_{h}-\hat{\boldsymbol{\tau}}\right) d \Omega \\
& =\int_{\Omega} \frac{\left|q_{h} \mathbf{I}+\hat{\boldsymbol{\tau}}\right|^{r^{\prime} / r-1}}{\left\|q_{h} \mathbf{I}+\hat{\boldsymbol{\tau}}\right\|_{T^{\prime}}^{r^{\prime}-1}}\left(q_{h} \mathbf{I}+\hat{\boldsymbol{\tau}}\right):\left(q_{h} \mathbf{I}+\hat{\boldsymbol{\tau}}\right) d \Omega-\int_{\Omega} \boldsymbol{\varsigma}_{h}:\left(\boldsymbol{\tau}_{h}-\hat{\boldsymbol{\tau}}\right) d \Omega \\
& \geq\left\|q_{h} \mathbf{I}+\hat{\boldsymbol{\tau}}\right\|_{T^{\prime}}-\left\|\boldsymbol{\varsigma}_{h}\right\|_{T}\left\|\boldsymbol{\tau}_{h}-\hat{\boldsymbol{\tau}}\right\|_{T^{\prime}} \\
& \geq\left(n^{1 / r^{\prime}}-1\right)\left\|q_{h}\right\|_{P}-2 \hat{C} h\left\|\operatorname{div} \boldsymbol{\tau}_{h}\right\|_{0, r^{\prime}, \Omega} .
\end{aligned}
$$

Let

$$
\mathbf{u}^{*}=\frac{-\left(n^{1 / r^{\prime}}-1\right)\left|\operatorname{div} \boldsymbol{\tau}_{h}\right|^{r^{\prime} / r-1}}{\left\|\operatorname{div} \boldsymbol{\tau}_{h}\right\|_{0, r^{\prime}, \Omega}^{r^{\prime}-1}}\left(\operatorname{div} \boldsymbol{\tau}_{h}\right),
$$

and note that $\left\|\mathbf{u}^{*}\right\|_{U}=n^{1 / r^{\prime}}-1$. Recall that $\operatorname{div} \boldsymbol{\psi}_{h} \in U_{h}$ for all $\boldsymbol{\psi}_{h} \in T_{d i v, h}^{\prime}$. Thus, there is a $\mathbf{v}_{h} \in U_{h}$ such that

$$
\int_{\Omega} \mathbf{v}_{h} \cdot \mathbf{w}_{h} d \Omega=\int_{\Omega} \mathbf{u}^{*} \cdot \mathbf{w}_{h} d \Omega, \quad \forall \mathbf{w}_{h} \in U_{h}
$$

with $\mathbf{v}_{h} \in U_{h}$ and $\left\|\mathbf{v}_{h}\right\|_{U} \leq\left\|\mathbf{u}^{*}\right\|_{U}=n^{1 / r^{\prime}}-1$. Let $\eta_{h}=0$, then we have

$$
\begin{aligned}
{\left[\mathbf{C}\left(\boldsymbol{\tau}_{h}, q_{h}\right),\left(\mathbf{v}_{h}, \eta_{h}\right)\right] } & =\left(n^{1 / r^{\prime}}-1\right) \int_{\Omega} \frac{\left|\operatorname{div} \boldsymbol{\tau}_{h}\right|^{r^{\prime} / r-1}}{\left\|\operatorname{div} \boldsymbol{\tau}_{h}\right\|_{0, r^{\prime}, \Omega}^{r^{\prime}-1}}\left(\operatorname{div} \boldsymbol{\tau}_{h}\right) \cdot\left(\operatorname{div} \boldsymbol{\tau}_{h}\right) d \Omega \\
& =\left(n^{1 / r^{\prime}}-1\right)\left\|\operatorname{div} \boldsymbol{\tau}_{h}\right\|_{0, r^{\prime}, \Omega} .
\end{aligned}
$$

Thus, from (4.35) and (4.36), we have

$$
\begin{aligned}
& \frac{\left[\mathbf{B}\left(\boldsymbol{\varsigma}_{h}\right),\left(\boldsymbol{\tau}_{h}, q_{h}\right)\right]+\left[\mathbf{C}\left(\boldsymbol{\tau}_{h}, q_{h}\right),\left(\mathbf{v}_{h}, \eta_{h}\right)\right]}{\left\|\left(\boldsymbol{\varsigma}_{h}, \mathbf{v}_{h}, \eta_{h}\right)\right\|_{T \times U \times \mathbb{R}}} \\
& \quad \geq \frac{1}{n^{1 / r^{\prime}}}\left(\left(n^{1 / r^{\prime}}-1\right)\left\|q_{h}\right\|_{P}+\left(n^{1 / r^{\prime}}-1-2 \hat{C} h\right)\left\|\operatorname{div} \boldsymbol{\tau}_{h}\right\|_{0, r^{\prime}, \Omega}\right),
\end{aligned}
$$

and, for $h$ small enough to satisfy $n^{1 / r^{\prime}}-1-2 \hat{C} h>0$, we have that

$$
\frac{\left[\mathbf{B}\left(\boldsymbol{\varsigma}_{h}\right),\left(\boldsymbol{\tau}_{h}, q_{h}\right)\right]+\left[\mathbf{C}\left(\boldsymbol{\tau}_{h}, q_{h}\right),\left(\mathbf{v}_{h}, \eta_{h}\right)\right]}{\left\|\left(\boldsymbol{\varsigma}_{h}, \mathbf{v}_{h}, \eta_{h}\right)\right\|_{T \times U \times \mathbb{R}}} \geq C\left\|\left(\boldsymbol{\tau}_{h}, q_{h}\right)\right\|_{T_{d i v}^{\prime} \times P},
$$

for some constant $C>0$.

$\underline{\text { Case 2: }}\left(\left\|\boldsymbol{\tau}_{h}\right\|_{T_{d i v}^{\prime}} \geq\left\|q_{h}\right\|_{P}\right)$

Let

$$
\tilde{\boldsymbol{\tau}}=\hat{\boldsymbol{\tau}}-\frac{1}{n}\left(\int_{\Omega} \operatorname{tr}(\hat{\boldsymbol{\tau}}) d \Omega\right) \mathbf{I}, \quad \boldsymbol{\tau}^{0}=\tilde{\boldsymbol{\tau}}-\frac{1}{n} \operatorname{tr}(\tilde{\boldsymbol{\tau}}) \mathbf{I}, \quad \boldsymbol{\phi}^{*}=\frac{-\left|\boldsymbol{\tau}^{0}\right|^{r^{\prime} / r-1}}{\left\|\boldsymbol{\tau}^{0}\right\|_{T^{\prime}}^{r^{\prime}-1}} \boldsymbol{\tau}^{0}
$$


and $\varsigma_{h} \in T_{h}$ satisfying

$$
\int_{\Omega} \varsigma_{h}: \phi_{h} d \Omega=\int_{\Omega} \phi^{*}: \phi_{h} d \Omega, \quad \forall \phi_{h} \in T_{h} .
$$

Note that $\tilde{\boldsymbol{\tau}}, \boldsymbol{\tau}^{0} \in T_{h}$ and $\left\|\boldsymbol{\varsigma}_{h}\right\|_{T} \leq\left\|\boldsymbol{\phi}^{*}\right\|_{T}=1$. Let

$$
\mathbf{u}^{*}=\frac{-2\left|\operatorname{div} \boldsymbol{\tau}_{h}\right|^{r^{\prime} / r-1}}{\left\|\operatorname{div} \boldsymbol{\tau}_{h}\right\|_{0, r^{\prime}, \Omega}^{r^{\prime}-1}}\left(\operatorname{div} \boldsymbol{\tau}_{h}\right)
$$

and $\mathbf{v}_{h} \in U_{h}$ be given by

$$
\int_{\Omega} \mathbf{v}_{h} \cdot \mathbf{w}_{h} d \Omega=\int_{\Omega} \mathbf{u}^{*} \cdot \mathbf{w}_{h} d \Omega, \quad \forall \mathbf{w}_{h} \in U_{h} .
$$

Observe that $\left\|\mathbf{v}_{h}\right\|_{U} \leq\left\|\mathbf{u}^{*}\right\|_{U}=2$. Let

$$
\eta_{h}=\operatorname{sgn}\left(\int_{\Omega} \operatorname{tr}\left(\boldsymbol{\tau}_{h}\right) d \Omega\right)\left(1+\frac{1}{C_{0}}\right) n^{-1 / 2}|\Omega|^{1 / r^{\prime}} .
$$

Then we have

$$
\begin{aligned}
& {\left[\mathbf{B}\left(\varsigma_{h}\right),\left(\boldsymbol{\tau}_{h}, q_{h}\right)\right]=\int_{\Omega} \frac{\left|\boldsymbol{\tau}^{0}\right|^{r^{\prime} / r-1}}{\left\|\boldsymbol{\tau}^{0}\right\|_{T^{\prime}}^{r^{\prime}-1}} \boldsymbol{\tau}^{0}: \boldsymbol{\tau}_{h} d \Omega} \\
& =\int_{\Omega} \frac{\left|\boldsymbol{\tau}^{0}\right|^{r^{\prime} / r-1}}{\left\|\boldsymbol{\tau}^{0}\right\|_{T^{\prime}}^{r^{\prime}-1}} \boldsymbol{\tau}^{0}: \tilde{\boldsymbol{\tau}} d \Omega-\int_{\Omega} \frac{\left|\boldsymbol{\tau}^{0}\right|^{r^{\prime} / r-1}}{\left\|\boldsymbol{\tau}^{0}\right\|_{T^{\prime}}^{r^{\prime}-1}} \boldsymbol{\tau}^{0}:\left(\tilde{\boldsymbol{\tau}}-\boldsymbol{\tau}_{h}\right) d \Omega \\
& \geq \int_{\Omega} \frac{\left|\boldsymbol{\tau}^{0}\right|^{r^{\prime} / r-1}}{\left\|\boldsymbol{\tau}^{0}\right\|_{T^{\prime}}^{r^{\prime}-1}} \boldsymbol{\tau}^{0}: \boldsymbol{\tau}^{0} d \Omega-\left\|\boldsymbol{\varsigma}_{h}\right\|_{T}\left\|\tilde{\boldsymbol{\tau}}-\boldsymbol{\tau}_{h}\right\|_{T^{\prime}} \\
& \geq\left\|\boldsymbol{\tau}^{0}\right\|_{T^{\prime}}-\left\|\tilde{\boldsymbol{\tau}}-\boldsymbol{\tau}_{h}\right\|_{T^{\prime}} \\
& \geq \frac{1}{C_{0}}\left\|\boldsymbol{\tau}_{h}\right\|_{T^{\prime}}-\left(1+\frac{\hat{C}\left(1+C_{0}+|\Omega|\right)}{C_{0}} h\right)\left\|\operatorname{div} \boldsymbol{\tau}_{h}\right\|_{0, r^{\prime}, \Omega} \\
& -\frac{n^{-1 / 2}|\Omega|^{1 / r^{\prime}}}{C_{0}}\left|\int_{\Omega} \operatorname{tr}\left(\boldsymbol{\tau}_{h}\right) d \Omega\right| \\
& -\hat{C}(1+|\Omega|) h\left\|\operatorname{div} \boldsymbol{\tau}_{h}\right\|_{0, r^{\prime}, \Omega}-n^{-1 / 2}|\Omega|^{1 / r^{\prime}}\left|\int_{\Omega} \operatorname{tr}\left(\boldsymbol{\tau}_{h}\right) d \Omega\right| \\
& =\frac{1}{C_{0}}\left\|\boldsymbol{\tau}_{h}\right\|_{T^{\prime}}-\left(1+\frac{\hat{C} h\left(1+2 C_{0}+\left(1+C_{0}\right)|\Omega|\right)}{C_{0}}\right)\left\|\operatorname{div} \boldsymbol{\tau}_{h}\right\|_{0, r^{\prime}, \Omega} \\
& -\left(1+\frac{1}{C_{0}}\right) n^{-1 / 2}|\Omega|^{1 / r^{\prime}}\left|\int_{\Omega} \operatorname{tr}\left(\boldsymbol{\tau}_{h}\right) d \Omega\right|,
\end{aligned}
$$

and

$$
\begin{aligned}
{\left[\mathbf{C}\left(\boldsymbol{\tau}_{h}, q_{h}\right),\left(\mathbf{v}_{h}, \eta_{h}\right)\right]=\int_{\Omega} } & \frac{2\left|\operatorname{div} \boldsymbol{\tau}_{h}\right|^{r^{\prime} / r-1}}{\left\|\operatorname{div} \boldsymbol{\tau}_{h}\right\|_{0, r^{\prime}, \Omega}^{r^{\prime}}}\left(\operatorname{div} \boldsymbol{\tau}_{h}\right) \cdot\left(\operatorname{div} \boldsymbol{\tau}_{h}\right) d \Omega \\
& +\left(1+\frac{1}{C_{0}}\right) n^{-1 / 2}|\Omega|^{1 / r^{\prime}}\left|\int_{\Omega} \operatorname{tr}\left(\boldsymbol{\tau}_{h}\right) d \Omega\right| \\
=2\left\|\operatorname{div} \boldsymbol{\tau}_{h}\right\|_{0, r^{\prime}, \Omega} & +\left(1+\frac{1}{C_{0}}\right) n^{-1 / 2}|\Omega|^{1 / r^{\prime}}\left|\int_{\Omega} \operatorname{tr}\left(\boldsymbol{\tau}_{h}\right) d \Omega\right|
\end{aligned}
$$


and

$$
\left\|\boldsymbol{\varsigma}_{h}\right\|_{T}+\left\|\mathbf{v}_{h}\right\|_{U}+\left|\eta_{h}\right|=3+\left(1+\frac{1}{C_{0}}\right) n^{-1 / 2}|\Omega|^{1 / r^{\prime}}=\tilde{C} .
$$

Thus, (4.38)-(4.40) and $h$ small enough to guarantee that

$$
C_{0}>\hat{C} h\left(1+2 C_{0}+\left(1+C_{0}\right)|\Omega|\right)
$$

imply

$$
\begin{aligned}
& \frac{\left[\mathbf{B}\left(\boldsymbol{\varsigma}_{h}\right),\left(\boldsymbol{\tau}_{h}, q_{h}\right)\right]+\left[\mathbf{C}\left(\boldsymbol{\tau}_{h}, q_{h}\right),\left(\mathbf{v}_{h}, \eta_{h}\right)\right]}{\left\|\left(\boldsymbol{s}_{h}, \mathbf{v}_{h}, \eta_{h}\right)\right\|_{T \times U \times \mathbb{R}}} \\
& \geq \frac{1}{\tilde{C} C_{0}}\left(\left\|\boldsymbol{\tau}_{h}\right\|_{T^{\prime}}\right. \\
& \left.\quad+\left(C_{0}-\hat{C} h\left(1+2 C_{0}+\left(1+C_{0}\right)|\Omega|\right)\right)\left\|\operatorname{div} \boldsymbol{\tau}_{h}\right\|_{0, r^{\prime}, \Omega}\right) \\
& \geq C\left\|\boldsymbol{\tau}_{h}\right\|_{T_{d i v}^{\prime}} \geq \frac{C}{2}\left\|\left(\boldsymbol{\tau}_{h}, q_{h}\right)\right\|_{T_{d i v}^{\prime} \times P} .
\end{aligned}
$$

Thus (4.34) is shown.

From the standard approximation properties $[9,7]$, the following error estimate is derived.

Theorem 4.3 Let $\mathbf{f} \in\left(L^{r^{\prime}}(\Omega)\right)^{2}$ and $\mathbf{u}_{\Gamma} \in\left(W^{1-1 / r, r}(\Gamma)\right)^{2}$. Let $(\boldsymbol{\phi}, \boldsymbol{\psi}, p, \mathbf{u}, \lambda) \in T \times T_{\text {div }}^{\prime} \times P \times U \times \mathbb{R}$ solve (2.13)-(2.15) and let $\left(\boldsymbol{\phi}_{h}, \boldsymbol{\psi}_{h}, p_{h}, \mathbf{u}_{h}, \lambda_{h}\right) \in T_{h} \times T_{\text {div }, h}^{\prime} \times P_{h} \times U_{h} \times \mathbb{R}$ solve (4.2)-(4.4). Assume $1 \leq m \leq k+1$ and $(\boldsymbol{\phi}, \boldsymbol{\psi}, p, \mathbf{u}) \in\left(W^{m, r}(\Omega)\right)^{2 \times 2} \times\left(W^{m, r^{\prime}}(\Omega)\right)^{2 \times 2} \times W^{m, r^{\prime}}(\Omega) \times\left(W^{m, r}(\Omega)\right)^{2}$ with $\operatorname{div} \boldsymbol{\psi} \in\left(W^{m, r^{\prime}}(\Omega)\right)^{2}$. Then there exists a positive constant $C$ such that

$$
\begin{gathered}
\left\|\boldsymbol{\phi}-\boldsymbol{\phi}_{h}\right\|_{T}^{2} \leq C\left\{h^{m r} \mathcal{E}\left(\boldsymbol{\phi}, \boldsymbol{\phi}_{h}\right)^{r}\|\boldsymbol{\phi}\|_{m, r, \Omega}^{r}\right. \\
\left.+h^{2 m}\left(\|\boldsymbol{\phi}\|_{m, r, \Omega}+\|\mathbf{u}\|_{m, r, \Omega}+\|\boldsymbol{\psi}\|_{m, r^{\prime}, \Omega}+\|\operatorname{div} \boldsymbol{\psi}\|_{m, r^{\prime}, \Omega}+\|p\|_{m, r^{\prime}, \Omega}\right)\right\} \\
\left\|\boldsymbol{\psi}-\boldsymbol{\psi}_{h}\right\|_{T_{d i v}^{\prime}}+\left\|p-p_{h}\right\|_{P} \leq C h^{m}\left(\|\boldsymbol{\psi}\|_{m, r^{\prime}, \Omega}+\|\operatorname{div} \boldsymbol{\psi}\|_{m, r^{\prime}, \Omega}+\|p\|_{m, r^{\prime}, \Omega}\right) \\
+\mathcal{E}\left(\boldsymbol{\phi}, \boldsymbol{\phi}_{h}\right)\left(\int_{\Omega}\left|\mathbf{g}(\boldsymbol{\phi})-\mathbf{g}\left(\boldsymbol{\phi}_{h}\right)\right|\left|\boldsymbol{\phi}-\boldsymbol{\phi}_{h}\right| d \Omega\right)^{1 / r^{\prime}} \\
\left\|\mathbf{u}-\mathbf{u}_{h}\right\|_{U}+\left|\lambda-\lambda_{h}\right| \leq C\left\|\boldsymbol{\phi}-\boldsymbol{\phi}_{h}\right\|_{T} .
\end{gathered}
$$

Remark 4.3 The extension of Remark 4.1 to these approximation spaces is given by: If $1 /(|\phi|+$ $\left.\left|\phi_{h}\right|\right) \leq C$ for some constant $C>0$ and $\left\|\phi-\phi_{h}\right\|_{\infty} \sim\left\|\phi-\phi_{h}\right\|_{T}$, the estimates (4.42)-(4.44) may be written as

$$
\begin{aligned}
\left\|\boldsymbol{\phi}-\boldsymbol{\phi}_{h}\right\|_{T}+\| \boldsymbol{\psi} & -\boldsymbol{\psi}_{h}\left\|_{T_{d i v}^{\prime}}+\right\| p-p_{h}\left\|_{P}+\right\| \mathbf{u}-\mathbf{u}_{h} \|_{U}+\left|\lambda-\lambda_{h}\right| \\
& \leq C h^{m}\left\{\|\boldsymbol{\phi}\|_{m, r, \Omega}+\|\mathbf{u}\|_{m, r, \Omega}+\|\boldsymbol{\psi}\|_{m, r^{\prime}, \Omega}+\|\operatorname{div} \boldsymbol{\psi}\|_{m, r^{\prime}, \Omega}+\|p\|_{m, r^{\prime}, \Omega}\right\} .
\end{aligned}
$$




\section{$5 \quad$ Numerical Experiments}

In this section we describe numerical experiments that support the theoretical results outlined in Sections 3 and 4. The first example illustrates the theoretical rate of convergence of the solution method and the second example illustrates the computed approximation for a benchmark physical problem. Computations are performed using the FreeFEM++ finite element software package [19]. All computations below are performed in the lowest-order case $(k=0)$.

\subsection{Example 1}

For this example (similar to one in [16]) approximations are computed for a Ladyzhenskaya law fluid with $\nu_{0}=0$ and $\nu_{1}=1.0$. The computational domain is $\Omega=[0,2] \times[0,2]$, with $\mathbf{f}$ and $\mathbf{u}_{\Gamma}$ chosen so that the exact solution of $(2.10)-(2.12)$ is given by

$$
\mathbf{u}=\left[\begin{array}{l}
u_{1} \\
u_{2}
\end{array}\right] \quad \text { and } \quad p=x_{1}+x_{2}
$$

with

$$
u_{1}=-\left(4.0-x_{1}-x_{2}\right)^{\alpha} \quad \text { and } \quad u_{2}=-u_{1}
$$

for $\alpha$ just large enough to ensure $\mathbf{f}=-\operatorname{div} \boldsymbol{\psi} \in W^{\mu-\varepsilon, r^{\prime}}(\Omega)$. It should be noted that $\alpha=-\frac{2}{r}+r^{\prime}+$ $\frac{\mu}{r-1}+\varepsilon$ ensures $\mathbf{f} \in W^{\mu, r^{\prime}}(\Omega)$ for $\varepsilon>0$.

Computations are performed on uniform meshes of decreasing size $h$ and for selected values of $r, \alpha$, and $\mu$. For $1<r<2$, the resulting system of equations is nonlinear, and a fixed-point iteration is used to compute approximations. The fixed-point iteration is terminated when the pointwise maximum absolute difference in successive approximations falls below $10^{-5}$. Results for the velocity, $\mathbf{u}$, the gradient of the velocity, $\phi(=\nabla \mathbf{u})$, and the total stress, $\boldsymbol{\psi}$, are shown in Table 5.1 .

For this example, $\operatorname{div} \psi \in W^{\mu-\varepsilon, r^{\prime}}(\Omega)$ is the most singular of the quantities to be approximated. The observed experimental convergence rate for $\left\|\operatorname{div} \boldsymbol{\psi}-\operatorname{div} \boldsymbol{\psi}_{h}\right\|_{0, r^{\prime}}$ of $C h^{\mu}$ is in agreement with that predicted by (4.16). The experimental convergence rates observed for $\left\|\boldsymbol{\phi}-\boldsymbol{\phi}_{h}\right\|_{T}$ and $\left\|\mathbf{u}-\mathbf{u}_{h}\right\|_{U}$ are both better than that given by (4.16).

\subsection{Example 2}

This example is the benchmark driven cavity problem. Driven cavity flows of power law fluids were computed using a mixed method by Manouzi and Farhloul in [23]. (In [23] the authors explicitly inverted the constitutive equation to obtain $\Phi_{\alpha}(\boldsymbol{\sigma})=\nabla \mathbf{u}$, which was used in their formulation.)

For $\Omega=[0,1] \times[0,1]$, we have that $\mathbf{f}=\mathbf{0}$ in $\Omega, \mathbf{u}_{\Gamma}=\mathbf{0}$ on $\Gamma \backslash \Gamma_{\text {top }}$ and $\mathbf{u}_{\Gamma}=\left[\begin{array}{ll}1 & 0\end{array}\right]^{\mathrm{T}}$ on $\Gamma_{\text {top }}$, where $\Gamma_{\text {top }}$ is the portion of the boundary satisfying $0 \leq x_{1} \leq 1$ and $x_{2}=1$. Computations were performed for a power law fluid with $\nu_{0}=1.0$ and selected values of $r$. Figures 5.1, 5.2, and 5.3 show plots of the streamlines computed for $h=1 / 32$ for $r=2, r=1.5$, and $r=1.1$, respectively. As the power $r$ in the constitutive law is decreased, we see a movement of the central vortex toward the top of the cavity, corresponding to an increase in viscosity. 


\begin{tabular}{|c|c||cc|cc|cc|}
\hline & $h$ & $\left\|\phi-\phi_{h}\right\|_{0, r}$ & rate & $\|$ div $\boldsymbol{\psi}-\operatorname{div} \boldsymbol{\psi}_{h} \|_{0, r^{\prime}}$ & rate & $\left\|\mathbf{u}-\mathbf{u}_{h}\right\|_{0, r}$ & rate \\
\hline \hline & 1 & 2.5481 & & 0.8014 & & 37.3797 & \\
$r=3 / 2$ & $1 / 2$ & 1.2633 & 1.01 & 0.4459 & 0.85 & 19.6284 & 0.93 \\
$\mu=1$ & $1 / 4$ & 0.6218 & 1.02 & 0.2426 & 0.88 & 9.8677 & 0.99 \\
$\alpha=11 / 3$ & $1 / 8$ & 0.3080 & 1.01 & 0.1299 & 0.90 & 4.9294 & 1.00 \\
& $1 / 16$ & 0.1534 & 1.01 & 0.0687 & 0.92 & 2.4623 & 1.00 \\
\hline & 1 & 1.3341 & & 0.2556 & & 10.5023 & \\
$r=3 / 2$ & $1 / 2$ & 0.6899 & 0.95 & 0.1824 & 0.49 & 5.3111 & 0.98 \\
$\mu=1 / 2$ & $1 / 4$ & 0.3405 & 1.02 & 0.1294 & 0.49 & 2.6503 & 1.00 \\
$\alpha=8 / 3$ & $1 / 8$ & 0.1677 & 1.02 & 0.0917 & 0.50 & 1.3223 & 1.00 \\
& $1 / 16$ & 0.0832 & 1.01 & 0.0648 & 0.50 & 0.6605 & 1.00 \\
\hline & 1 & 2.6967 & & 1.3410 & & 4721.1800 & \\
$r=5 / 4$ & $1 / 2$ & 1.3109 & 1.04 & 0.7234 & 0.89 & 2553.9800 & 0.89 \\
$\mu=1$ & $1 / 4$ & 0.6325 & 1.05 & 0.3833 & 0.92 & 1285.9000 & 0.99 \\
$\alpha=37 / 5$ & $1 / 8$ & 0.3094 & 1.03 & 0.2007 & 0.93 & 635.6200 & 1.02 \\
& $1 / 16$ & 0.1533 & 1.01 & 0.1042 & 0.95 & 315.0940 & 1.01 \\
\hline & 1 & 1.4671 & & 0.1661 & & 363.2130 & \\
$r=5 / 4$ & $1 / 2$ & 0.7461 & 0.98 & 0.1176 & 0.50 & 191.1110 & 0.93 \\
$\mu=1 / 2$ & $1 / 4$ & 0.3604 & 1.05 & 0.0832 & 0.50 & 94.7585 & 1.01 \\
$\alpha=27 / 5$ & $1 / 8$ & 0.1746 & 1.05 & 0.0588 & 0.50 & 46.8215 & 1.02 \\
& $1 / 16$ & 0.0860 & 1.02 & 0.0416 & 0.50 & 23.2479 & 1.01 \\
\hline
\end{tabular}

Table 5.1: Approximation errors and rates of convergence for Example 1.

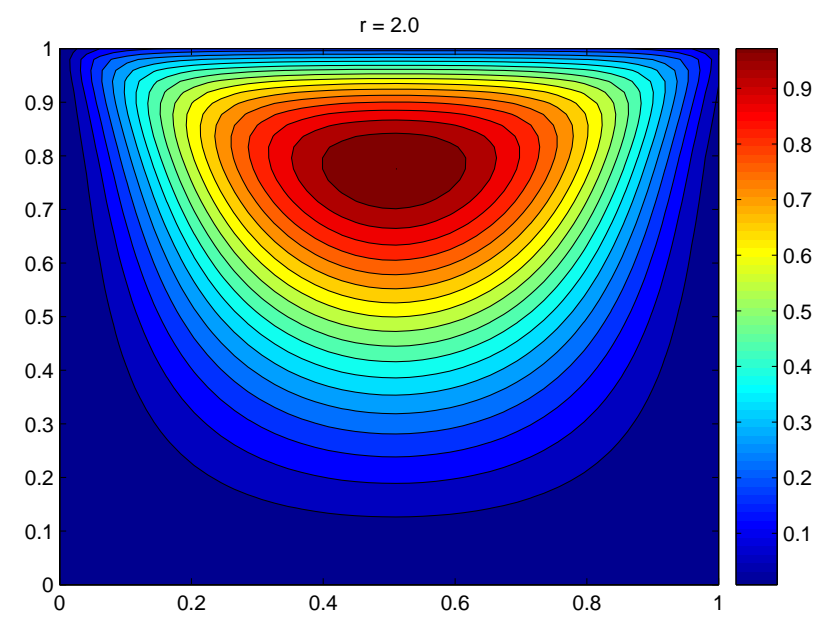

Figure 5.1: Streamlines for $r=2.0$, driven cavity

\section{References}

[1] R.A. Adams and J.J.F. Fournier, Sobolev Spaces, vol. 140 of Pure and Applied Mathematics, Academic Press, 2003. 


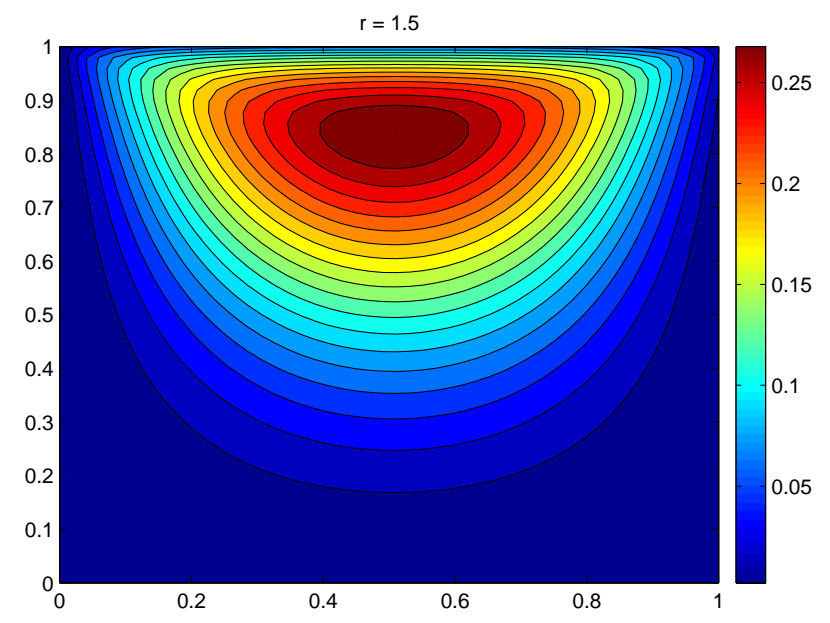

Figure 5.2: Streamlines for $r=1.5$, driven cavity

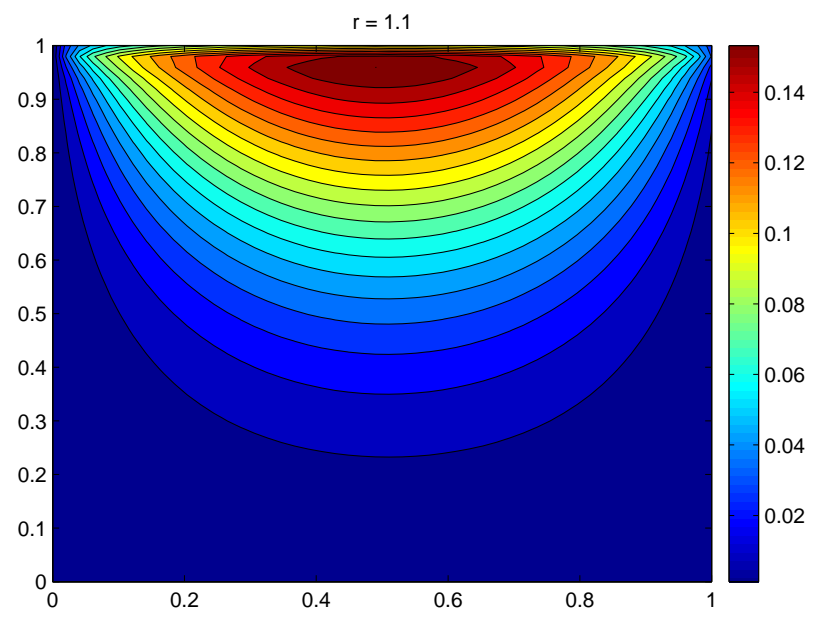

Figure 5.3: Streamlines for $r=1.1$, driven cavity

[2] D.N. Arnold, J. Douglas Jr. and C.P. Gupta, A family of higher order mixed finite element methods for plane elasticity, Numer. Math., 45, 1-22, 1984.

[3] J. Baranger and K. Najib, Analyse numerique des ecoulements quasi-Newtoniens dont la viscosite obeit a la loi puissance ou la loi de Carreau, Numer. Math., 58, 35-49, 1990.

[4] J. Baranger, K. Najib and D. Sandri, Numerical analysis of a three-fields model for a quasiNewtonian flow, Comput. Methods Appl. Mech. Engrg., 109, 281-292, 1993.

[5] R.B. Bird, R.C. Armstrong, and O. Hassager, Dynamics of Polymeric Liquids, John Wiley and Sons, New York, 1987.

[6] S.C. Brenner and L.R. Scott, The Mathematical Theory of Finite Element Methods, SpringerVerlag, 1994. 
[7] F. Brezzi and M. Fortin, Mixed and Hybrid Finite Element Methods, Springer-Verlag, New York, 1991.

[8] P.G. Ciarlet, The Finite Element Method for Elliptic Problems, SIAM, 2002.

[9] A. Ern and J.-L. Guermond, Theory and Practice of Finite Elements, Springer-Verlag, 2004.

[10] V.J. Ervin and J.S. Howell and I. Stanculescu, A dual-mixed approximation method for a three-field model of a nonlinear generalized Stokes problem, Clemson University Department of Mathematical Sciences Technical Report TR2007_08_EHS, 2007.

http://www.math.clemson.edu/reports/TR2007_08_EHS.pdf

[11] V.J. Ervin and H. Lee, Numerical approximation of a quasi-Newtonian Stokes flow problem with defective boundary conditions, to appear, SIAM J. Numer. Anal..

[12] V.J. Ervin and T.N. Phillips, Residual a posteriori error estimator for a three-field model of a non-linear generalized Stokes problem, Comput. Methods Appl. Mech. Engrg., 195, 2599-2610, 2006.

[13] M. Farhloul and H. Manouzi, On a mixed finite element method for the $p$-Laplacian, Canad. Appl. Math. Quart., 8, 67-78, 2000.

[14] G.P. Galdi, An Introduction to the Mathematical Theory of the Navier-Stokes Equations, Vol. 1, Springer-Verlag, New York, (1994).

[15] G.N. Gatica, M. Gonzalez, and S. Meddahi, A low-order mixed finite element method for a class of quasi-Newtonian Stokes flows. Part I: a priori error analysis, Comput. Methods Appl. Mech. Engrg., 193, 881-892, 2004.

[16] G.N. Gatica, M. Gonzalez, and S. Meddahi, A low-order mixed finite element method for a class of quasi-Newtonian Stokes flows. Part II: a posteriori error analysis, Comput. Methods Appl. Mech. Engrg., 193, 893-911, 2004.

[17] V. Girault and P.A. Raviart, Finite element methods for Navier-Stokes equations, SpringerVerlag, Berlin, Heidelberg, 1986.

[18] C.D. Han, Multiphase Flow in Polymer Processing, Academic Press, New York, 1981.

[19] F. Hecht, A. LeHyaric, and O. Pironneau. Freefem++ version 2.12-1, 2007. http://www.freefem.org/ff++.

[20] E. Kreyszig, Introductory Functional Analysis with Applications, Wiley Classics Library, John Wiley \& Sons Inc., New York, 1989.

[21] O.A. Ladyzhenskaya, New equations for the description of the viscous incompressible fluids and solvability in the large of the boundary value problems for them, Boundary Value Problems of Mathematical Physics V, American Mathematical Society, Providence RI, (1970).

[22] P.D. Lax, Functional Analysis, John Wiley and Sons, New York, 2002.

[23] H. Manouzi and M. Farhloul, Mixed finite element analysis of a non-linear three-fields Stokes model, IMA J. Numer. Anal., 21, 143-164, 2001. 
[24] R.G. Owens and T.N. Phillips, Computational Rheology, Imperial College Press, London, 2002.

[25] P.A. Raviart and J.M. Thomas, A mixed finite element method for 2-nd order elliptic problems, Lecture notes in Math., 606, 292-315, 1977.

[26] M. Renardy and R.C. Rogers, An Introduction to Partial Differential Equations Springer, New York, 1993.

[27] J.E. Roberts and J.M. Thomas, Mixed and hybrid finite element methods, in Handbook of Numerical Analysis, Vol. II, Finite element methods, Part 1 (P. G. Ciarlet and J. L. Lions, eds.), North Holland, Amsterdam, 1989.

[28] D. Sandri, On the numerical approximation of quasi-Newtonian flows whose viscosity obeys a power law or the Carreau law, RAIRO Modél. Math. Anal. Numér., 27, 131-155, 1993.

[29] D. Sandri, A posteriori estimators for mixed finite element approximations of a fluid obeying the power law, Comput. Methods Appl. Mech. Engrg., 166, 329-340, 1998. 